\title{
AS REVISÕES SISTEMÁTICAS COMO FONTES DE EVIDÊNCIAS NAS RECOMENDAÇÕES DE SAÚDE: O CASO DA AMAMENTAÇÃO E A SAÚDE DA CRIANÇA
}

\author{
Martha Silvia Martinez-Silveira \\ Fundação Oswaldo Cruz. Instituto de Comunicação e Informação Científica e Tecnológica em \\ Saúde. Instituto Gonçalo Moniz \\ martha.silveira@gmail.com
}

Cícera Henrique da Silva Instituto de Comunicação e Informação Cintífica e Tecnológica em Saúde; Fundação Oswaldo Cruz - Rio de Janeiro, RJ

Josué Laguardia Instituto de Comunicação e Informação Cintífica e Tecnológica em Saúde; Fundação Oswaldo Cruz - Rio de Janeiro, RJ

\begin{abstract}
Resumo
As revisões sistemáticas são uma síntese metodologicamente construída a partir dos estudos originais consideradas eficientes geradoras de evidências científicas para as decisões de saúde, porém sua qualidade pode interferir limitando sua utilização. Sua importância se percebe pela crescente produção e utilização nas recomendações de saúde. Tais recomendações baseadas em evidências científicas, elaboradas por instituições de reconhecida influência, orientam o comportamento sobre as ações de saúde. O objetivo deste estudo foi investigar as revisões sistemáticas como fontes de evidências científicas das recomendações sobre o tema, as repercussões da amamentação na saúde da criança. Se fez uma análise de citação para identificar as revisões sistemáticas referenciadas nas recomendações de instituições internacionais como o Brasil, Estados Unidos e Canadá. A qualidade metodológica das revisões foi avaliada usando Amstar. Foram selecionadas 101 recomendações que citaram 30 revisões sistemáticas. As revisões são de qualidade moderada e ainda insuficientes quanto à temática e aos achados, que foram escassamente citadas
\end{abstract}

Palavras-chave: Resiliência. Evidência cientifica. Revisão sistemática. Recomendações de saúde. Qualida-de. Aleitamento materno.

\section{SYSTEMATIC REVIEWS AS SOURCES OF EVIDENCE IN HEALTH RECOMMENDATIONS: THE CASE OF BREASTFEEDING AND CHILD HEALTH}

\begin{abstract}
Systematic reviews are considered effective in generating high-level scientific evidence for health care decisions. However, their quality can impact its usefulness. Their importance can be seen in their increasing use in medical practice guidelines and health recommendations. Such recommendations based on scientific evidence, drawn up by recognized institutions, influence and guide behaviors on health-related actions. The objective was to investigate systematic reviews as source of scientific evidence in the recommendations about the effects of breastfeeding on the child's health. It was a citation analysis to identify systematic reviews referenced in the recommendations of international institutions, as well as institutions from Brazil, United States and Canada. The methodological quality of the reviews was assessed using Amstar. We selected 101 recommendations and 30 systematic reviews cited. systematic reviews. They were barely mentioned in the recommendations, are of moderate quality. Only few reviews became citation pattern, they are not current neither conclusive.
\end{abstract}

Keywords: Scientific evidence. Systematic review. Health Recommendation. Quality. Breast Feeding.

\section{INTRODUÇÃO}

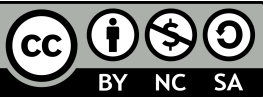

P2P \& INOVAÇÃO, Rio de Janeiro, v. 5 n. 2, p.133-174, Mar./Ago. 2019. 
Abordar o tema de evidências científicas em saúde requer uma aproximação ao complexo processo de tomada de decisão onde algo mais que a apropriação das evidências científicas está em jogo (LIMIEUX-CHARLES; CHAMPAGNE, 2004). Trata-se de um processo político e social para o qual não se pode afirmar que o uso de evidências produz necessariamente melhores decisões (LOMAS, 2004), embora estas devessem ser o alicerce de qualquer decisão que envolve a saúde. As pesquisas científicas são a fonte e base das evidências científicas. Estas, por seu turno, são oriundas do resultado da aplicação de um método de experimentação, observação ou exploração de um fenômeno, cujos dados, quantitativos e/ou qualitativos, são coletados, analisados e interpretados pelos cientistas (LOMAS et al., 2005). Entretanto, estudos sobre evidências científicas apontam que elas são fragmentadas, provisórias e muitas vezes, conflitivas e que isto dificulta a sua apropriação nas práticas e nos processos decisórios de saúde. A despeito das decisões acerca de políticas e programas de saúde poderem estar situadas para além dos estudos científicos (LOMAS, 2004), estes são fonte de informação indispensável ao processo.

A produção de evidências científicas orientadas à assistência e às políticas e recomendações de saúde tem origem em diversas fontes que geram informação necessária para a tomada de decisão e poderão ter diferentes formatos, a depender do conteúdo e do demandante ou potencial usuário. À medida que a implementação de práticas informadas por evidências avança, percebe-se um olhar mais amplo sobre os tipos de estudos que geram evidências. Titler (2008) ressalta que a melhor evidência vem dos estudos clínicos randomizados e da pesquisa qualitativa. No entanto, há um consenso generalizado sobre a validade de estudos secundários, especificamente as revisões sistemáticas, não somente para produção de evidências, mas também para uma melhor efetivação do processo de transferência dos resultados da pesquisa para sua utilização nas instâncias decisórias (LAVIS et al., 2003).

A revisão sistemática (RS) é um método de síntese eficiente na geração de evidências científicas a partir dos estudos originais, cujos resultados são a informação de mais alto nível científico (HIGGINS, 2011; MEERPOHL et al., 2012). É também um meio de obter respostas sobre o que funciona e o que não funciona, sobre a efetividade ou o custo-benefício de uma intervenção (PETTICREW; ROBERTS, 2006). O método da RS procura reunir resultados de estudos primários para aumentar a qualidade das evidências obtidas por meio das amostras fracionadas de cada estudo (HEMINGWAY; BRERETON, 2009). A importância das RS está associada à necessidade de se obter evidências mais sólidas para os diversos procedimentos da prática médica e para o desenvolvimento de programas e políticas de saúde pública (GREENHALGH, 2008; GUYATT et al., 2011a). As RS combinam os achados de vários 
estudos menores, criam resultados mais robustos e convincentes e podem provar as inconsistências ou a falta de evidências apontando a necessidade de mais e melhores estudos primários (PETTICREW; ROBERTS, 2006; GUYATT et al., 2011a).

Sua importância é destacada na crescente produção e utilização de evidências em documentos que guiam as práticas médicas e as políticas e recomendações de saúde (WHO, 2012; BRASIL, 2012). As recomendações de saúde são definidas pela Organização Mundial da Saúde (OMS) como todo tipo de documento que contenha recomendações sobre intervenções de saúde, tanto da área clínica, de saúde pública ou política pública de saúde. São o produto das instâncias decisórias de instituições públicas ou privadas. As recomendações fornecem informações sobre o que os decisores políticos, os prestadores de cuidados de saúde ou pacientes devem fazer (WHO, 2012), orientando o comportamento sobre as ações de saúde, tanto da sociedade quanto dos indivíduos, em particular. Dessa forma estes documentos passam a ter um caráter oficial, o que levou à decisão de nomeá-las, neste artigo, como recomendações oficiais de saúde (RO). Sabe-se também que estas recomendações devem estar baseadas em evidências científicas, como indicam as diretivas do Ministério da Saúde (BRASIL, 2006; 2012), e por este motivo elas foram escolhidas para a análise nesta pesquisa. Diante do exposto, o pressuposto que orientou o presente trabalho é o de que as RS são fontes indispensáveis e obrigatórias de evidências para as RO, e que assim sendo, é esperado que incluam em seu texto as referências onde se encontram tais evidências. Todavia, a simples citação de uma RS não garante que uma RO seja cientificamente bem fundamentada, interessa saber a qualidade dessa RS e quais foram suas conclusões e limitações de forma a categorizar os trabalhos científicos que geram evidências e apoiam as recomendações de saúde.

A avaliação da qualidade de um estudo pode ser entendida como um processo que trata de estabelecer quão próximo da "verdade" seus achados têm probabilidade de estar e se esses estudos são de relevância para um determinado assunto (CRD, 2009). Avaliar a qualidade de uma revisão significa, de modo geral, levar em consideração aspectos relevantes, tais como a seleção dos estudos, a avaliação da qualidade dos estudos, a combinação dos dados dos estudos e suas conclusões, entre outros (SHEA et al., 2007). A importância de cada um desses aspectos vai depender do foco e da natureza da avaliação, embora se saiba que a avaliação da qualidade dos estudos produz melhores níveis de confiança dos achados científicos (LOHR, 2004). Esta pesquisa avaliou a qualidade metodológica da RS por meio do uso de um instrumento validado, o Assessment of Multiple Systematic Reviews (AMSTAR) (SHEA et al., 2009)

O tema, repercussões da amamentação na saúde da criança, foi escolhido por tratar-se de uma questão de saúde pública que envolve controvérsias que demanda de muitos estudos. $\mathrm{O}$ ato 
de amamentar representa uma decisão comportamental, preferencialmente da mãe, mas também do entorno familiar, porém as repercussões desse ato são de interesse da saúde pública. As pesquisas nesse tópico enfrentam dificuldades para mensurar aspectos que se referem ao comportamento humano, à influência contextual sobre a individualidade e como estes se combinam em fatores que confundem, enviesam os resultados e enfraquecem as evidências. $\mathrm{O}$ interesse pela amamentação na literatura científica é profuso, atual e com uma quantidade significativa de publicações que mostram as inúmeras implicações biomédicas e comportamentais do ato de amamentar e do seu produto, o leite humano, embora nem todo artigo represente uma evidência. É provável que esse volume também decorra dos diferentes olhares multidisciplinares da ciência, assim como também as incertezas que circundam o tema. Há um consenso, aparentemente consolidado na literatura, em torno do aleitamento materno e seus efeitos positivos à saúde da criança (WHO, 2007; 2010; BRASIL; OPAS, 2002). Na literatura mais atual percebe-se um direcionamento a produzir evidências sobre a efetividade de programas de promoção da amamentação e no seu prolongamento. Os países em estudo, além do Brasil, foram escolhidos com o intuito de efetuar comparações; o Canadá por seu perfil de modelo na produção e uso de evidências e os Estados Unidos como tradicional fonte de pesquisas em saúde que embasam as ciências da saúde no Brasil.

Dessa forma, o objetivo deste trabalho é verificar se as RS são fontes de evidências científicas das RO do Brasil, Canadá e Estados Unidos e organizações internacionais, sobre os efeitos da amamentação na saúde da criança e qual é sua qualidade metodológica.

\section{MÉTODOS}

Trata-se de um estudo exploratório com análise de citação, no qual foi realizada uma busca abrangente das RO sobre amamentação produzidas no Brasil, Canadá, Estados Unidos e por instituições internacionais de saúde, com a análise das citações de revisões sistemáticas presentes nestas recomendações. Não foi colocado nenhum limite quanto à data de publicação.

\subsection{Identificação das Instituições e classificação das instituições}

Para realizar a busca das RO, o processo começou pela identificação das possíveis instituições que as emitiriam. Assim, a busca de instituições e órgãos oficiais representativos e reconhecidos na área da saúde dos três países foi realizada na web a partir da ferramenta Google e acesso aos sites, em diferentes etapas e por países. 
As instituições foram classificadas em quatro categorias: a) Associação profissional de saúde, incluindo associações, sociedades, academias e colégios de especialistas relativos ao tema; b) Instituição governamental de saúde, incluindo ministérios, secretarias de saúde, departamentos, instituições e agências do governo; c) Organismo internacional de saúde; d) Organização filantrópica independente de saúde incluindo aqui instituições civis com finalidades de interesse público que não tem fins lucrativos, e atuam de forma independentes do governo e que estivessem vinculadas ao tema e produzissem material científico para profissionais de saúde.

\subsection{Seleção das Recomendações}

Quanto ao conteúdo das RO, tomou-se como modelo para seleção o artigo de Burda, Chambers e Johnson (2014), que analisaram as recomendações da OMS e os tipos de documentos tidos como recomendações. Dessa forma, os documentos foram selecionados e categorizados em: recomendação, política, declaração, posicionamento, parecer, guia, diretriz, protocolo, consenso, manual, capítulo de livro e conteúdo para cursos e relatórios técnicos, relativos ao tema e com possível influência direta na atuação do profissional de saúde, policymakers ou instituições de saúde, excluindo os documentos elaborados exclusivamente para o público em geral (Quadro 1).

Quadro 1 - Tipo de documentos considerados como Recomendações oficiais

\begin{tabular}{|c|c|c|}
\hline TIPO & INGLÊS & DEFINIÇÃOO \\
\hline Política & $\begin{array}{l}\text { Policy/ } \\
\text { Policy } \\
\text { statement }\end{array}$ & 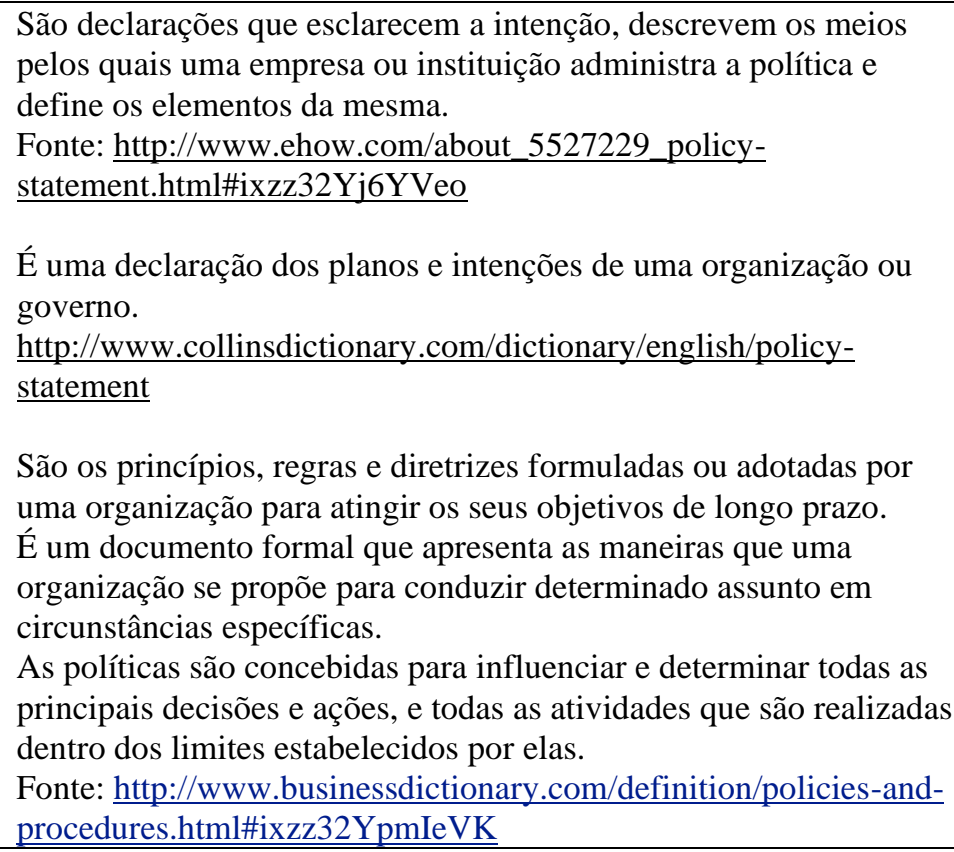 \\
\hline $\begin{array}{l}\text { Declaração/ } \\
\text { Posicionamento/ } \\
\text { Parecer }\end{array}$ & $\begin{array}{l}\text { Position or } \\
\text { Positioning } \\
\text { Statement/ }\end{array}$ & $\begin{array}{l}\text { Posicionamento ou declaração oficial institucional ou } \\
\text { organizacional a respeito de um tema. } \\
\text { Opinião, recomendação base, declarações. }\end{array}$ \\
\hline
\end{tabular}




\begin{tabular}{|c|c|c|}
\hline & $\begin{array}{l}\text { Joint } \\
\text { Statement }\end{array}$ & $\begin{array}{l}\text { A declaração ou parecer é uma explicação, uma justificativa ou } \\
\text { uma recomendação para um curso de ação que reflete a posição em } \\
\text { relação ao assunto. Declarações de posição expiram ou são } \\
\text { abandonados pela organização, quando apropriado. } \\
\text { Fonte: http://www.nursingworld.org/positionstatements }\end{array}$ \\
\hline $\begin{array}{l}\text { Recomendações/ } \\
\text { Guias/Diretrizes/ } \\
\text { Orientações/ } \\
\text { Protocolos/ } \\
\text { Consensos }\end{array}$ & $\begin{array}{l}\text { Guidelines/ } \\
\text { Consensus } \\
\text { statement }\end{array}$ & $\begin{array}{l}\text { Diretrizes são orientações ou princípios que apresentam regras } \\
\text { atuais ou futuras da política para ajudar os profissionais de saúde } \\
\text { nas decisões de atendimento ao paciente quanto ao diagnóstico, } \\
\text { terapia ou circunstâncias clínicas relacionadas e de todos os } \\
\text { aspectos dos cuidados e assistência à saúde. } \\
\text { Protocolos são planos precisos e detalhados para o estudo de um } \\
\text { problema médico ou biomédico e / ou planos para um regime de } \\
\text { terapia. } \\
\text { Consensos são declarações que representam o acordo da maioria } \\
\text { dos médicos, cientistas e outros profissionais convocados com a } \\
\text { finalidade de alcançar um consenso - muitas vezes com conclusões } \\
\text { e recomendações - em um assunto de interesse. } \\
\text { Fonte: Medical Subject Headings (MeSH) } \\
\text { http://www.ncbi.nlm.nih.gov/mesh } \\
\text { Qualquer documento que contém uma recomendação para o uso de } \\
\text { uma intervenção de saúde, seja clínica, de saúde pública ou } \\
\text { recomendaçôes políticas } \\
\text { Fonte: World Health Organization. WHO handbook for guideline } \\
\text { development. Geneva: World Health Organization; } 2012 . \\
\text { Para a OMS, recomendação e guideline referem-se ao mesmo tipo } \\
\text { de documento. }\end{array}$ \\
\hline $\begin{array}{l}\text { Relatório } \\
\text { Técnico/ } \\
\text { Nota Técnica/ } \\
\text { Posicionamento } \\
\text { técnico/ } \\
\text { Revisão } \\
\text { sistemática }\end{array}$ & $\begin{array}{l}\text { Special } \\
\text { Report/ } \\
\text { Summary } \\
\text { Report/ } \\
\text { Technical } \\
\text { statement }\end{array}$ & $\begin{array}{l}\text { Nota técnica, Relatório Especial, Relatório Técnico; Revisão } \\
\text { Sistemática encomendada por uma instituição de saúde para } \\
\text { embasar/orientar decisões. Este tipo de documento tem que ter uma } \\
\text { instituição como autora ou responsável pela publicação. } \\
\text { Relatório técnico - Trabalho que consiste em relatório formal dando } \\
\text { detalhes da pesquisa e dos resultados de um problema médico ou } \\
\text { científico. Quando publicada por um órgão governamental ou } \\
\text { agência oficial, seu conteúdo pode ser secreto ou não, de acordo } \\
\text { com as medidas de segurança. Este tipo de publicação também } \\
\text { pode cobrir um artigo científico ou o estado atual ou posição atual } \\
\text { de uma pesquisa científica e seu desenvolvimento. Se o editor lhe } \\
\text { der este rótulo, este tipo de publicação pode ser adequadamente } \\
\text { utilizado em trabalhos publicados em periódicos. } \\
\text { Fonte: Descritores em Ciências da Saúde (DeCS) - } \\
\text { http://decs.bvs.br: }\end{array}$ \\
\hline $\begin{array}{l}\text { Manuais/ } \\
\text { Capítulos para } \\
\text { livros/ } \\
\text { Apostilas para } \\
\text { cursos/ }\end{array}$ & $\begin{array}{l}\text { Manual/ } \\
\text { Chart } \\
\text { booklet/ } \\
\text { Model } \\
\text { chapter for } \\
\text { textbooks/ } \\
\text { Tool or } \\
\text { Toolkit/ } \\
\text { Operation } \\
\text { Manual }\end{array}$ & $\begin{array}{l}\text { Manuais, cursos, capítulos de livro e material elaborado para servir } \\
\text { de apoio a técnicos e profissionais de saúde. }\end{array}$ \\
\hline
\end{tabular}

Fonte: Elaborado pelos autores (2018)

Os documentos localizados foram selecionados por dois avaliadores de forma independente e cega (MSMS, CHS) com base nos seguintes critérios de inclusão: a) Material de nível científico destinado aos profissionais da área de saúde; b) tema de amamentação e suas 
implicações da saúde da criança, podendo abranger todo ou apenas uma parte do documento, em cujo caso levou-se em consideração apenas o capítulo do tema de interesse; c) autoria de uma instituição de acordo com a categorização acima mencionada. Nos casos de discordância o item foi reavaliado para o consenso, caso contrário, um terceiro avaliador foi contatado.

\subsection{Análise de citação}

Foram analisadas de forma manual as referências bibliográficas de cada RO para identificar as RS citadas nos documentos segundo os seguintes critérios: a) constar da lista de referências do capítulo, do documento como um todo ou no pé de página, b) menção no título da referência dos termos revisão sistemática, revisão, metanálise, análise conjunta, revisão de evidências ou similares, c) se no título ficasse claro tratar-se do tema amamentação e criança. Em caso de dúvida a referência era selecionada e conferida através do acesso à base de dados; caso não fosse suficiente, o texto completo era acessado.

Além disso, foi verificado também se havia citação de outras recomendações e menção a possíveis instituições produtoras de recomendações sobre o tema.

\subsection{Características das RS citadas}

Todas as RS citadas que tratassem do tema foram selecionadas e a qualidade avaliada segundo Amstar por dois revisores independentes (MSMS, JL). Os dados extraídos foram: tema, classificado segundo o vocabulário controlado Descritores em Ciências da Saúde (DeCS); país, número e desenho dos estudos incluídos, se houve avaliação da qualidade dos estudos e nome da ferramenta utilizada, se houve metanálise, principais conclusões e limitações reportadas, número de vezes que foi citada por alguma das recomendações. As conclusões foram analisadas a partir dos efeitos da amamentação. $O$ relato sobre os efeitos manteve na medida do possível o texto original, assim foram identificados os efeitos positivos, negativos ou nenhum efeito na saúde da criança. A magnitude do efeito reportado foi também extraída e classificada nas seguintes categorias: a) alto, substancial ou significativo, b) modesto, c) pequeno ou fraco e d) possível.

Quanto às limitações, criaram-se as seguintes categorias de análise: a) reporta necessidade de novos estudos, incluindo-se aqui falta de dados ou dados insuficientes, necessidade de dados de mais qualidade, necessidade de melhores estudos, mais amplos e mais atuais; b) reporta falhas metodológicas dos estudos originais, incluindo-se aqui: viés de memória, falta de definição do conceito "amamentação exclusiva", falta de definição da doença em estudo, não 
ajuste de fatores de confundimento no estudo, falhas e distorções na agrupação de casos e controles; c) reporta não efetuar ajuste de fatores de confundimento na RS; reporta heterogeneidade entre os estudos da RS; e) reporta viés de publicação na RS; f) reporta viés de seleção na RS; g) reporta não aplicabilidade dos resultados da RS.

A pesquisa foi dispensada de avalição pelo Comité de Ética em Pesquisa da Escola Politécnica de Saúde Joaquim Venâncio, da Fundação Oswaldo Cruz. Não há conflito de interesses e nem houve financiamento nesta pesquisa.

\section{RESULTADOS}

As buscas por RO resultaram em 164 possíveis documentos, dos quais foram selecionados 101, sendo que 26 eram brasileiros, 32 norte-americanos, 7 canadenses e 36 elaborados por instituições internacionais de saúde (APÊNDICE B). A maioria das RO do Brasil (96\%) foi publicada pelo Ministério da Saúde e apenas uma por associação profissional (4\%). As RO canadenses foram publicadas por instituições do governo $(28,6 \%)$, por associações profissionais $(42,8 \%)$ ou por ambas em conjunto $(28,6 \%)$. As RO norte-americanas foram publicadas em sua maioria por associações profissionais $(56,3 \%)$, e instituições do governo (25\%). Individualmente, a instituição que mais publicou foi a OMS, com 23 documentos, seguida do Ministério da Saúde do Brasil, com 22. É importante destacar que as RO foram localizadas nas buscas na web e um grande número nas listas de referencias das próprias $\mathrm{RO}$.

Quanto ao tipo, 48 (47,5\%) documentos foram classificados como recomendação; 21 $(20,8 \%)$ como manual e material para incluir em livros ou cursos para profissionais da área de saúde; 19 (18,8\%) como declaração de posicionamento da instituição; 8 (7,9\%) como política pública de saúde e 5 (5\%) como relatório de especialistas para embasar decisões de saúde.

Foram contabilizadas 8.638 referências nas $101 \mathrm{RO}$, dentre as quais foram identificadas 115 RS (1,3\%) que atendiam aos critérios de inclusão. Uma vez excluídas as duplicadas, restaram $30 \mathrm{RS}$ diferentes (Tabela 1 e Apêndice C) que representavam 0,35\% do total de referências das RO. Das 101 RO, 19 (18,8\%) não tinham nenhuma referência em seu texto. As RO classificadas como recomendações foram, no total, as que tinham mais referências, responsáveis por $52 \%$ das citações, e referenciaram $34 \%$ das RS citadas. No conjunto das 82 RO que tinham referências, $40(48,8 \%)$ não citaram nenhuma RS relativa ao tema. Dentre as 42 que citaram, 20 correspondiam a recomendações; 11 a manuais e materiais para cursos e livros; 5 a declarações, 3 a políticas e 3 a relatórios técnicos. 
Uma RO emitida por uma instituição governamental dos Estados Unidos, publicada em 2007 e classificada como relatório técnico (AHRQ, 2007) foi a que mais citou RS (16), seguida de outra emitida pela OMS e classificada com manual ou material para cursos ou capítulos de livros (WHO, 2009), que citou 11 RS.

O período de publicação das RO é bastante extenso (1984 a 2013) e verificou-se que aquelas que citaram são mais recentes (2000-2013) quando comparadas às que nada citaram. Até o ano de 2007, apenas 21 RS tinham sido citadas em 10 RO, uma média de 2,1 RS por RO. De 2007 em diante 93 RS foram citadas em 31 RO, uma média de 3 RS por RO.

Ao analisar segundo o tipo de documento, observou-se que as RO classificadas como "recomendação" foram as que mais citaram RS (40), seguidas dos manuais e materiais para cursos e livros que citaram 29 RS, dos relatórios técnicos que citaram 24 RS, das declarações que citaram 12 RS e das políticas que citaram 10.

A RS mais citada foi a de Kramer e Kakuma (2002a) (Amstar 7), que trata do tempo de duração ótima para amamentação exclusiva, com 15 citações. Esta revisão possui diversas versões, foi publicada pela OMS (KRAMER; KAKUMA, 2002a) e como revisão da Cochrane (KRAMER, M. S.; KAKUMA, 2002b) no mesmo ano, porém com uma atualização dos estudos incluídos na versão original. Posteriormente foi republicada em um periódico (KRAMER; KAKUMA, 2004) e teve duas atualizações em 2007 (disponível na base da Cochrane até 2009) e em 2012 (KRAMER; KAKUMA, 2009; 2012), onde foram incluídos novos estudos. Se consideradas todas as versões e atualizações, esta RS foi citada por $22 \mathrm{RO}$ diferentes desde 2002 até 2013, quase ininterruptamente, em todas suas versões. As outras RS mais citadas foram a de Horta et al. (2007) (Amstar 6), sobre os efeitos da amamentação a longo prazo e a de Anderson, Johnstone e Remley (1999) (Amstar 3), sobre desenvolvimento cognitivo e amamentação, ambas com 12 citações e a de Arenz et al. (2004) (Amstar 7), sobre obesidade e amamentação que teve 8 citações (Tabela 1).

Das 26 diferentes RS, 4 (16,7\%) utilizaram apenas ensaios clínicos randomizados, 4 $(16,7 \%)$ apenas estudos de coorte e $5(20,8 \%)$ somente casos controle, destas todas tinham metanálise. A metade (13) das RS (50\%) utilizou uma combinação de diferentes estudos, sendo mais frequente a combinação de estudos de coorte com caso-controle e estudos transversais, sendo que 5 RS $(19,2 \%)$ combinaram estudos randomizados e controlados com estudos de coorte e estudos transversais.

Mais de $40 \%$ das RS citadas não efetuaram uma avaliação da qualidade dos estudos originais incluídos nas revisões, sendo que todas tinham a metanálise. Quando avaliados, os critérios mais utilizados foram os Critérios da Cochrane (23\%) (Tabela 1). 
Dentre os 13 grupos temáticos gerados, houve uma prevalência de Obesidade e sobrepeso (24\%), seguida de Hipertensão e colesterol (20\%) e Doenças intestinais (20\%) (Tabela 2).

Tabela 2 - Grupos temáticos entre as RS citadas

\begin{tabular}{lcc}
\hline \multirow{2}{*}{ Grupo temático } & \multicolumn{2}{c}{ RS citadas } \\
\hline Obesidade; Sobrepeso & n & \% \\
Doenças Intestinais & 6 & 24 \\
Hipertensão; Colesterol & 5 & 20 \\
Crescimento; Desenvolvimento & 5 & 20 \\
Inteligência; Cognição & 4 & 16 \\
Diabetes Mellitus & 4 & 16 \\
Doenças do Sistema Imunológico & 3 & 12 \\
Morbidade; Mortalidade Infantil & 3 & 12 \\
Cárie dentária; Má oclusão & 2 & 8 \\
Dor/prevenção e controle & 2 & 8 \\
Infecções; Doenças Transmissíveis & 2 & 8 \\
Neoplasias & 2 & 8 \\
Doenças Metabólicas & 1 & 4 \\
\hline
\end{tabular}

Fonte: Elaborado pelos autores (2018)

A avaliação com o Amstar mostrou que 5 (16,6\%) tinham baixa qualidade ( 0 -4 pontos) e $25(83,3 \%)$ qualidade média (5-8 pontos). Na análise individual observou-se que somente 5 RS completaram $72,7 \%$ dos itens do Amstar enquanto 12 completaram aproximadamente a metade dos itens $(54,4 \%)$. Houve 1 RS que não completou nenhum dos itens. Há uma concentração na nota 6 , de qualidade média, como se observa na figura 1 .

Figura 1- Resultado da avaliação com Amstar das RS citadas

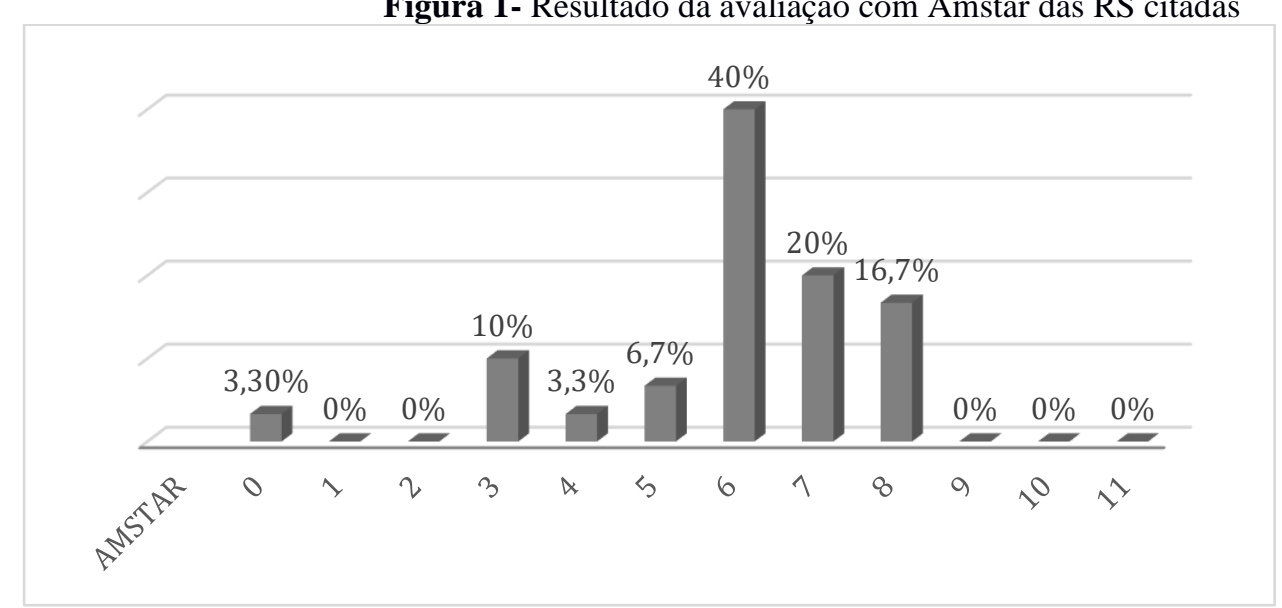

Fonte: Elaborado pelos autores (2018)

As falhas metodológicas mais frequentes foram a ausência de informação sobre a existência ou não de conflito de interesse nos estudos incluídos nas RS (100\%), a não realização de um protocolo anterior à realização da $\mathrm{RS}(86,7 \%)$ e a não complementação das buscas dos 
estudos na literatura cinzenta $(53,3 \%)$. Por outro lado, os itens mais frequentemente presentes nas RS avaliadas foram a apresentação de uma tabela ou relato com as características dos estudos incluídos $(93,3 \%)$ e a combinação apropriada de estudos $(83,3 \%)$ e avaliação dos estudos incluídos (60\%). Dentre as 67 RS selecionadas, 30 foram citadas nas RO.

Quanto às limitações, na maioria das RS $(65,4 \%)$ os autores declararam que havia necessidade de serem realizados novos e melhores estudos ou que não havia dados suficientes, e em mais da metade das RS foram reportadas falhas metodológicas dos estudos originais (53\%). As RS que reportaram tais limitações foram confrontadas com o resultado obtido no item do Amstar sobre terem ou não efetuado avaliação da qualidade dos estudos, no sentido de averiguar como os autores chegaram a essas limitações, os resultados mostram que quase a metade $(48,8 \%)$ não tinha feito a avaliação, e a maioria $(81,4 \%)$ obteve qualificação média de acordo com o Amstar (Tabela 3).

Tabela 3 - Principais limitações das RS reportadas pelos autores

\begin{tabular}{lccccc}
\hline \multicolumn{1}{c}{ Limitações } & \multicolumn{2}{c}{$\begin{array}{c}\text { Avalição da } \\
\text { Qualidade }\end{array}$} & Amstar & \multicolumn{2}{c}{ Total } \\
\cline { 2 - 6 } & Sim & Não & B & M & n (\%) \\
\hline Necessidade de novos estudos & 9 & 8 & 3 & 14 & $17(65,0)$ \\
Falhas metodológicas dos estudos originais & 7 & 7 & 3 & 11 & $14(53,0)$ \\
Não efetuar ajuste para fatores de confundimento & 2 & 3 & 1 & 4 & $5(19,2)$ \\
Heterogeneidade entre os estudos & 1 & 1 & 0 & 2 & $2(7,7)$ \\
Não aplicabilidade dos resultados & 2 & 0 & 0 & 2 & $2(7,7)$ \\
Viés de publicação & 0 & 2 & 0 & 2 & $2(7,7)$ \\
Viés de seleção & 1 & 0 & 1 & 0 & $1(3,8)$ \\
B= baixa qualidade (notas 0-4); M= qualidade média (notas 5-8) & & & &
\end{tabular}

Fonte: Elaborado pelos autores (2018)

Quanto às evidências reportadas, estas foram analisadas levando em conta apenas os estudos únicos (26) e que cada estudo pode avaliar mais de um aspecto. Identificaram-se 25 tópicos diferentes, sendo que para cada tópico podia haver mais de um estudo e consequentemente mais de um efeito (Quadro 2). Em 11 desses tópicos a AM teve um efeito positivo sobre alguns aspectos da saúde da criança, mas a magnitude foi reportada como moderada ou pequena em 5 casos, significativa ou forte em 3 tópicos e possível ou não reportada em 3 casos. Na hipertensão, por exemplo, os efeitos podem ser protetores, reduzir o risco ou provocarem uma pequena redução nos níveis tensionais, porém a magnitude é pequena ou modesta. Em quatro tópicos o efeito da AM foi considerado negativo pelos estudos, sendo que a magnitude foi considerada fraca ou pequena em 3 deles e forte em apenas um. Verificaramse 12 tópicos avaliados por mais de um estudo e os efeitos reportados para um mesmo tópico foram diferentes em 8 casos. Na dermatite atópica, por exemplo, os efeitos podem ser protetores 
de forma significativa ou pode não haver efeito algum. No desenvolvimento cognitivo, assim como a avaliação da inteligência e cognição, os efeitos são reportados como significativos, ou muito pequenos ou ainda nenhum efeito foi encontrado (Quadro 2).

Quadro 2 - Análise das conclusões dos autores segundo efeitos da amamentação na saúde da criança como reportados nas RS.

\begin{tabular}{|c|c|c|c|}
\hline \multirow{2}{*}{$\begin{array}{l}\text { Aspectos da saúde da } \\
\text { criança estudados (autor e } \\
\text { ano) }\end{array}$} & \multirow{2}{*}{ Efeitos reportados } & \multicolumn{2}{|c|}{ Classificação } \\
\hline & & Tipo & Magnitude \\
\hline $\begin{array}{l}\text { Asma na infância } \\
\text { (Gdalevich 2001a) } \\
\text { (Kramer 2002a) }\end{array}$ & $\begin{array}{l}\text { Menores taxas nas crianças AM exclusiva no } 1^{\circ} \\
\text { mês } \\
\text { AM exclusiva } 6 \text { meses não reduz o risco }\end{array}$ & $\begin{array}{l}\text { Positivo } \\
\text { Nenhum }\end{array}$ & Forte \\
\hline $\begin{array}{l}\text { Cárie dentária precoce } \\
\text { (Valaitis 2000) } \\
\text { (Kramer 2002a) }\end{array}$ & $\begin{array}{l}\text { AM por } 1 \text { ano à noite pós dentição está } \\
\text { associada. } \\
\text { AM exclusiva } 6 \text { meses não reduz o risco }\end{array}$ & $\begin{array}{l}\text { Negativo } \\
\text { Nenhum }\end{array}$ & Moderada \\
\hline $\begin{array}{l}\text { Colesterol } \\
\text { (Horta 2007) } \\
\text { (Owen 2007) }\end{array}$ & $\begin{array}{l}\text { Não efeito significativo em crianças e } \\
\text { adolescentes, efeito pequeno em adultos. } \\
\text { Média de CT e LDL maior em AM vs AF na } \\
\text { infância, sem diferença na adolescência e mais } \\
\text { baixo na vida adulta }\end{array}$ & $\begin{array}{l}\text { Nenhum } \\
\text { Positivo }\end{array}$ & Modesta \\
\hline $\begin{array}{l}\text { Dermatite atópica } \\
\text { (Gdalevich 2001b) } \\
\text { (Kramer 2002a) } \\
\end{array}$ & $\begin{array}{l}\text { Efeito protetor significativo AM exclusiva de } \\
\text { família com atopias. } \\
\text { AM exclusiva } 6 \text { meses não reduz o risco }\end{array}$ & $\begin{array}{l}\text { Positivo } \\
\text { Nenhum } \\
\end{array}$ & Significativa \\
\hline $\begin{array}{l}\text { Desenvolvimento infantil } \\
\text { (Kramer 2002a) }\end{array}$ & Não déficit por AM exclusiva & Positivo & Modesta \\
\hline $\begin{array}{l}\text { Desenvolvimento e } \\
\text { crescimento em PT BPN. } \\
\text { (Quigley 2007) } \\
\text { (Henderson 2001) }\end{array}$ & $\begin{array}{l}\text { Maiores taxas em AF vs LM doado no curto } \\
\text { prazo. } \\
\text { Maiores taxas AF } v s \text { LM de termo não } \\
\text { fortificada. }\end{array}$ & $\begin{array}{l}\text { Negativo } \\
\text { Negativo }\end{array}$ & $\begin{array}{l}\text { Forte } \\
\text { Forte }\end{array}$ \\
\hline $\begin{array}{l}\text { Desenvolvimento cognitivo } \\
\text { (Anderson 1999) } \\
\text { (Kramer 2002a) }\end{array}$ & $\begin{array}{l}\text { Maior desenvolvimento ( } 6 \text { a } 23 \text { meses). E maior } \\
\text { ainda em BPN. } \\
\text { AM exclusiva } 6 \text { meses não mostra influência }\end{array}$ & $\begin{array}{l}\text { Positivo } \\
\text { Nenhum }\end{array}$ & Significativa \\
\hline $\begin{array}{l}\text { Diabetes mellitus tipo } 1 \\
\text { (Norris 1996) }\end{array}$ & Risco pequeno de associação à AM & Negativo & Fraca \\
\hline $\begin{array}{l}\text { Diabetes mellitus tipo } 2 \\
\text { (Horta 2007) } \\
\text { (Owen 2006) }\end{array}$ & $\begin{array}{l}\text { Sem conclusões sobre os efeitos de longo prazo } \\
\text { de AM sobre o risco } \\
\text { Risco reduzido }\end{array}$ & $\begin{array}{l}\text { Nenhum } \\
\text { Positivo }\end{array}$ & Modesta \\
\hline $\begin{array}{l}\text { Doença celíaca } \\
\text { (Akobeng 2006) }\end{array}$ & Reduz significativamente o risco & Positivo & Significativa \\
\hline $\begin{array}{l}\text { Doença inflamatória intestinal } \\
\text { (Klement 2004) } \\
\text { (Barclay 2009) }\end{array}$ & $\begin{array}{l}\text { Menor risco } \\
\text { Possível proteção }\end{array}$ & $\begin{array}{l}\text { Positivo } \\
\text { Positivo }\end{array}$ & $\begin{array}{c}\text { Significativa } \\
\text { Possível }\end{array}$ \\
\hline $\begin{array}{l}\text { Doenças alérgicas } \\
\text { (Kramer 2002a) }\end{array}$ & AM exclusiva 6 meses não reduz o risco & Nenhum & \\
\hline $\begin{array}{l}\text { Doenças respiratórias } \\
\text { (Bachrach 2003) }\end{array}$ & Menor risco de hospitalização & Positivo & Forte \\
\hline $\begin{array}{l}\text { Enterocolite necrosante em } \\
\text { prematuros } \\
\text { (McGuire 2003) } \\
\text { (Quigley 2007) } \\
\text { (Henderson 2001) }\end{array}$ & $\begin{array}{l}\text { Menor incidência } \\
\text { Menor risco } \\
\text { Os dados não são conclusivos }\end{array}$ & $\begin{array}{l}\text { Positivo } \\
\text { Positivo } \\
\text { Nenhum }\end{array}$ & $\begin{array}{l}\text { Pequena } \\
\text { Forte }\end{array}$ \\
\hline $\begin{array}{l}\text { Hipertensão } \\
\text { (Martin 2005b) } \\
\text { (Owen 2003) } \\
\text { (Horta 2007) }\end{array}$ & $\begin{array}{l}\text { Efeito protetor } \\
\text { Pequena redução } \\
\text { Efeito protetor }\end{array}$ & $\begin{array}{l}\text { Positivo } \\
\text { Positivo } \\
\text { Positivo }\end{array}$ & $\begin{array}{l}\text { Pequena } \\
\text { Modesta } \\
\text { Pequena }\end{array}$ \\
\hline Infecções gastrointestinais & Risco reduzido AM exclusiva 6 meses ou + & & \\
\hline
\end{tabular}




\begin{tabular}{|c|c|c|c|}
\hline (Kramer 2002a) & & Positivo & Significativa \\
\hline $\begin{array}{l}\text { Infecções respiratórias } \\
\text { (Kramer 2002a) }\end{array}$ & Risco reduzido AM exclusiva 6 meses ou + & Positivo & Significativa \\
\hline $\begin{array}{l}\text { Inteligência e cognição } \\
\text { (Der 2006) } \\
\text { (Horta 2007) }\end{array}$ & $\begin{array}{l}\text { Efeito pequeno ou ausente } \\
\text { Pouco aumento }\end{array}$ & $\begin{array}{l}\text { Nenhum } \\
\text { Positivo }\end{array}$ & Pequena \\
\hline $\begin{array}{l}\text { Intolerância alimentar } \\
\text { (Henderson 2001) }\end{array}$ & Menor risco & Positivo & Pequena \\
\hline $\begin{array}{l}\text { Leucemia (L) } \\
\text { (Kwan 2004) }\end{array}$ & Protetor L. linfoblástica e L. mieloide & Positivo & Possível \\
\hline $\begin{array}{l}\text { Mortalidade por doenças } \\
\text { cardiovasculares (DCV) } \\
\text { (Matin 2004) }\end{array}$ & $\begin{array}{l}\text { Não influência, mas AM de longo prazo } \\
\text { aumenta } 16 \% \text { morte DCV }\end{array}$ & Negativo & Pequena \\
\hline $\begin{array}{l}\text { Morte súbita do lactente } \\
\text { (Hauck 2011) }\end{array}$ & Redução do risco e maior se for AM exclusiva & Positivo & NR \\
\hline $\begin{array}{l}\text { Nível de ferro } \\
(\text { Kramer 2002a) }\end{array}$ & Redução & Negativo & Fraca \\
\hline $\begin{array}{l}\text { Obesidade e sobrepeso } \\
\text { (Harder 2005) } \\
(\text { Horta 2007) } \\
(\text { Owen 2005) } \\
(\text { Arenz 2004) }\end{array}$ & $\begin{array}{l}\text { Diminui risco } \\
\text { Proteção } \\
\text { Diminui o risco } \\
\text { Efeito protetor de obesidade na infância }\end{array}$ & $\begin{array}{l}\text { Positivo } \\
\text { Positivo } \\
\text { Positivo } \\
\text { Positivo }\end{array}$ & $\begin{array}{l}\text { Pequena } \\
\text { Pequena } \\
\text { NR } \\
\text { Pequena }\end{array}$ \\
\hline $\begin{array}{l}\text { Procedimentos dolorosos em } \\
\text { neonatos } \\
\text { (Shah 2006) }\end{array}$ & Útil & Positivo & NR \\
\hline
\end{tabular}

$\mathrm{AM}=$ Amamentado; $\mathrm{AF}=$ Alimentação por fórmula; $\mathrm{PT}=$ Prematuro; $\mathrm{BPN}=$ Baixo peso ao nascer

Fonte: Elaborado pelos autores (2018)

\section{DISCUSSÃO}

As numerosas e variadas RO que formam parte deste estudo resultaram difíceis de serem localizadas e selecionadas. As instituições utilizam formatos e canais distintos e isso fica mais evidente na comparação entre os países. No Canadá, por exemplo, o Health Canada, que é o departamento federal de saúde, em concordância com as principais associações profissionais canadenses, emitiu uma declaração conjunta na qual se detalham os mais diversos aspectos da amamentação (HEALTH CANADA et al., 2014). Já nos Estados Unidos, existem muitas instituições profissionais em diferentes campos da saúde que emitem declarações, recomendações e pareceres e a tendência a se posicionar perante os temas de saúde fez com que a maioria dos documentos fosse classificada como "declaração" ou "Position Statement".

No Brasil falta uma padronização na emissão e divulgação das recomendações. O Ministério da Saúde brasileiro emitiu 24 dos 26 documentos selecionados, mas para identificar quais eram os pareceres do Ministério em relação à amamentação foi preciso incluir neste estudo manuais e material para cursos. As publicações brasileiras que foram classificadas como "recomendações" utilizam o formato de guias, porém não houve nenhum material que pudesse ser considerado como declaração ou posicionamento institucional a respeito do tema. 
A razão das datas destas RO não serem tão atuais pode se dever a que mais recentemente nota-se uma mudança na temática das recomendações, estas se direcionam ao incentivo para aumentar o número de mulheres que amamentam e sobre o apoio que devem receber, tanto institucional como social. Este fato parece sinalizar que as instituições responsáveis pelo direcionamento das questões de saúde da criança acreditam que as evidências em relação aos benefícios e repercussões da amamentação já estão mais consolidadas e, portanto, não haveria tanta preocupação em produzir recomendações. Todavia, os resultados observados nessa pesquisa questionam a solidez de tais evidências.

De acordo com a grande quantidade de recomendações encontradas e a quantidade de citações existentes nas RO avaliadas (mais de 8 mil), o número de citações de RS ainda é muito pequeno, a despeito da preocupação de que as recomendações se apoiem em evidências científicas (BURDA; CHAMBERS; JOHNSON, 2014). Isso não significa, não entanto, que as RO não estejam baseadas em evidências, uma vez que outros trabalhos também geram evidências, mas chama a atenção que quase $40 \%$ delas não citou nenhuma RS, em compensação foi muito significativo o número de RO que estavam citadas nas RO. Este tipo de resultado já foi reportado por Oxman, Lavis e Fretheim (2007), que destacaram que as recomendações da OMS faziam pouco uso das RS, prevalecendo a opinião de especialistas como forma de evidência. Uma das causas da escassa citação pode estar relacionada com falta de revisões sistemáticas disponíveis ou a escassez de temas abordados, mas é importante destacar que a qualidade destas revisões influencia bastante na possibilidade da sua citação.

O estudo com o Amstar indicou que a qualidade metodológica dessas revisões é essencialmente média, atingindo em sua maioria 6 pontos. A questão da qualidade de uma revisão e a qualidade dos estudos incluídos nessa revisão é de fundamental importância para a evidência que se reporta, uma vez que sua força dependerá dessa avaliação. Este "controle de qualidade" tem feito com que as RS e metanálises se tornassem os estudos científicos com o melhor nível de evidência para embasar as políticas e as decisões de saúde (PETTICREW; ROBERTS, 2006). O Amstar tem se mostrando eficiente para seu propósito em diversos estudos nacionais e internacionais e. seu uso permitiu visualizar que, de acordo aos resultados obtidos, as RS não estão atendendo aos critérios de qualidade, ou, ao menos seu relato não está refletindo uma qualidade ótima. Alguns itens estão frequentemente presentes nas RS, e outros frequentemente ausentes. A busca em bases de dados, por exemplo, é uma etapa que aparece bem relatada em uma grande quantidade de RS avaliadas. Entretanto, esta ferramenta não permite saber se essas buscas foram bem efetuadas, sendo comum as pesquisas que apontam falhas e dificuldades na busca dos estudos originais (SAMPSON et al., 2009), na utilização de 
estratégias (RELEVO, 2012) e a necessidade de integrar peritos em buscas às equipes de RS, tais como os bibliotecários (MARTÍNEZ-SILVEIRA, 2011).

As dificuldades na confecção de uma RS extrapolam o uso adequado da metodologia. Uma das limitações mais frequente é a inexistência de estudos primários que atendam os critérios mínimos de qualidade e especificidade; outras limitações têm origem nas falhas metodológicas dos estudos originais (PETTICREW et al., 1999) que impõem barreiras à produção de evidências e a heterogeneidade entre os estudos (FLETCHER, 2007). Nesta pesquisa, 53\% das RS citadas reportaram ter encontrado falhas metodológicas importantes nos estudos analisados, mas somente metade delas fez uma avaliação da qualidade dos estudos. Cerca de $40 \%$ dos estudos que a avaliaram, por sua vez, não fizeram a correlação ente a baixa qualidade dos estudos e as conclusões e recomendações. A heterogeneidade nos desenhos e a forma de utilizar os dados dos estudos originais impedem de realizar a metanálise. Entretanto, $75 \%$ das RS fizeram algum tipo de teste para avaliar a homogeneidade entre os estudos antes de combinar os dados, mas apenas 2 RS reportaram essa limitação. Disso se deduz que ou essa dificuldade foi superada ou, apesar da heterogeneidade, fez-se a análise dos dados, agrupandoos por tipo de estudo, o qual parece ser o mais provável, devido à grande combinação de desenhos de estudos detectados nas RS avaliadas.

A escassez de estudos originais e sua qualidade pode ter também relação com o tema do estudo, a amamentação, pois este possui características particulares que dificultam a produção de evidências por meio de estudos experimentais. Embora sejam considerados como o tipo de estudo mais eficiente para produzir evidências de maior nível (GUYATT et al., 2011b), estudos experimentais seriam antiéticos ao expor crianças a outro tipo de alimentação (WHO, 2000). Gdalevich e colaboradores (2001) ressaltam também que o principal fator que induz ao viés nos estudos desse tema é o fato da amamentação ser uma prática relacionada a uma escolha pessoal da mãe, que está sujeita a diversas influências subjetivas, sociais e culturais, que se convertem em fatores de confundimento de difícil mensuração. Outro problema apontado é o uso de uma definição vaga ou imperfeita dos fenômenos estudados, o que dificulta a obtenção de homogeneidade nos dados. Desse modo, ao se falar sobre amamentação exclusiva, torna-se difícil determinar ou saber se realmente foi exclusiva, pois podem ocorrer esquecimento ou mesmo desconhecimento de eventos que interromperam a amamentação. Por estas razões os estudos observacionais, a despeito de possuírem um menor nível de evidência devido à sua propensão aos vieses, foram os mais prevalentes nas revisões (LEVINE, 2011).

O número reduzido de temas abordados nas RS avaliadas neste estudo, que, agrupados por similaridade e interconexão com o auxílio do DeCS, representaram apenas 13 grupos, 
sinaliza a escassez de temas investigados e a dispersão e falta de aprofundamento nos achados, que se mostra insuficiente para reverter o quadro atual de um predomínio de resultados inconclusivos ou contraditórios observados nas RS avaliadas.

Quando analisadas as conclusões das RS citadas, observou-se uma predominância dos efeitos positivos da amamentação (60,9\%), trazendo à tona uma realidade presente na literatura da área de saúde onde é mais comum a publicação de resultados positivos (FRANCO; MALHOTRA; SIMONOVITS, 2014). Não obstante, há uma particularidade das RS que permite, ao fazer um levantamento o agrupamento dos resultados, mostrar que as evidências podem ser diferentes daquelas dos estudos originais ou mesmo tornar mais clara a falta de evidências. Dessa forma, verificou-se que em mais da metade dos aspectos estudados $(58,5 \%)$, os resultados apresentavam efeitos de magnitude pequenos, baixos, modestos ou mesmo nenhum efeito, enquanto que em $24,4 \%$ dos aspectos o efeito foi classificado como significativo ou forte, sem que isso represente, contudo, uma evidência forte, já que podem ser diversos os efeitos dentro de um mesmo tópico estudado, permanecendo a controvérsia.

A análise de citação revelou que o relatório técnico da Agency for Health Research and Quality (AHRQ) (AHRQ, 2007), dos Estados Unidos foi a RO que mais citou RS (16), seguida do modelo para capítulo de livro de estudantes e profissionais da saúde, editado em 2009 pela OMS (WORLD HEALTH ORGANIZATION, 2010), que citou 11 RS. A política sobre amamentação e leite humano da American Academy of Pediatrics publicada em 2012 citou 7 RS. Dentre estas, 5 foram as mesmas citadas pela duas RO anteriores. Com isto nota-se que a citação das RS permanece em uma espécie de círculo vicioso, onde duas ou três RS são citadas de forma contínua ao longo dos anos, e onde RO citam outras RO, especialmente as da OMS.

A RS mais citada sobre o tempo de duração da amamentação, editado pela OMS em 2002 (KRAMER; KAKUMA, 2002a), por exemplo, foi citada 15 vezes, sete das quais por RO da própria OMS, mas também aparece citada em RO do Canadá, dos EUA e do Brasil. Esta RS vem sendo citada nas RO, em quase todos os anos, desde 2002. As outras duas RS mais citadas (ANDERSON; JOHNSTONE; REMLEY, 1999; HORTA et al., 2007) também vem sendo citadas ao longo dos anos.

A recorrência de citação dessas RS pode estar relacionada à importância de seus achados e com o fato de serem as primeiras a abrangerem diversos aspectos dos efeitos da amamentação em um mesmo trabalho. Outra explicação se daria pelo fato delas terem sido encomendadas pela OMS para gerarem evidências que sustentassem a recomendação da amamentação em todo o mundo. O estudo de Anderson e colaboradores (ANDERSON; JOHNSTONE; REMLEY, 
1999) teve importância por ser a primeira metanálise que relacionou um efeito positivo da amamentação no desenvolvimento cognitivo das crianças e adolescentes estudados.

A RS de Kramer e Kakuma (2002a) procurou trazer evidências sobre os efeitos da amamentação exclusiva por seis meses no desenvolvimento e crescimento da criança. A visão de que o leite materno exclusivo fosse insuficiente para suprir as necessidades nutricionais do bebê, somada à ideia de que os alimentos dados como complemento são inadequados e passíveis de contaminação, colocou em cena o que se denominou do "dilema do desmame". No início dos anos 2000, as recomendações da OMS e da Unicef diferiam quanto ao que deveria ser considerado como duração ótima. Enquanto a OMS recomendava amamentação exclusiva de 4 a 6 meses e, logo após, a introdução de alimentos complementares, a Unicef não estipulava limites tão claros, aconselhando que a amamentação exclusiva fosse "por mais ou menos 6 meses". A Academy of Pediatrics, à época, trazia recomendações contraditórias em seu manual, pois recomendava o uso do leite materno exclusivo por seis meses em um dos capítulos e o adiamento da introdução de alimentos sólidos até os 4 ou 6 meses, em outro capítulo (AMERICAN ACADEMY OF PEDIATRICS apud KRAMER; KAKUMA, 2002a). Diante de tal situação, a OMS encomendou uma RS e com base nas evidências obtidas, tanto a OMS quanto a Unicef emitiram suas recomendações de amamentação exclusiva por seis meses e continuação da amamentação por dois anos ou mais. Esta revisão teve ampla divulgação e diversas atualizações, mas seus achados não se modificaram substancialmente.

Quanto a RS de Horta (2007) surge de um interesse generalizado em verificar os efeitos da amamentação a longo prazo, ou seja, no desenvolvimento intelectual e na vida adulta, uma vez que à época um estudo realizado em países de média e baixa renda havia relatado os efeitos a curto prazo, especificamente a redução da mortalidade e morbidade por doenças infecciosas (WORLD HEALTH ORGANIZATION, 2000), mas existiam controvérsias sobre as consequências da amamentação a longo prazo. Esse estudo trouxe evidências que sugeriam benefícios a longo prazo nos aspectos relativos à hipertensão, colesterol, obesidade, inteligência e DMT2.

\section{CONCLUSÕES}


Os achados deste estudo mostram que as revisões sistemáticas são pouco citadas nas recomendações, têm qualidade média em sua maioria e ainda são insuficientes para sustentar as evidências. Os assuntos estudados são limitados a poucos temas e as conclusões podem ser discordantes e indefinidas. Poucas RS se converteram em padrão de citação nas RO, isto é, RS citadas com mais frequência e por mais tempo nas RO, mas estas não são as mais atuais e nem conclusivas. Isto talvez explique o fato de haver novas revisões sendo publicadas sobre tais temáticas nos últimos anos, apesar de não serem citadas.

Os efeitos reais da amamentação sobre a saúde da criança são reportados em sua maioria como modestos ou pequenos, em muitos casos devido às falhas metodológicas verificadas nos estudos originais ou às limitações com que se defrontam os autores da RS. Contudo, as recomendações parecem considerar as evidências existentes como definitivas e suficientes para recomendar a amamentação em mais de 100 documentos de diferentes tipologias publicados em três países e por organismos internacionais de saúde. Possivelmente as evidências venham das revisões citadas, mas também de muitos outros estudos originais que podiam estar citados, mas que não foram objeto desta pesquisa.

Para Upshur (2000), as evidências médicas sofrem um contínuo processo de atualização e mudança, que é visto como o processo lógico do avanço científico acompanhado de novas descobertas. Os temas amamentação e saúde da criança não fogem à regra, com evidências provisórias, incompletas e emergentes. Dado que a utilização da evidência é colocada como um processo social e político (UPSHUR, 2000), a inclusão ou não de uma evidência em recomendações oficiais de saúde pode ser devido a questões distintas daquelas tradicionalmente inferidas quando avaliamos uma citação.

De acordo Nicolaisen (2007), a citação é uma forma de legitimar o conhecimento anterior e um compromisso ético e moral com quem anteriormente expressou conceitos, ideias e teorias que influenciaram o autor citante (NICOLAISEN, 2007). MacRoberts e MacRoberts (2010) apontam que os autores citam apenas partes das suas influências, o que levaria a pressupor que as citadas são consideradas por eles como mais importantes. Citar também pode ser um ato de persuasão, quando o autor de certa forma manipula os documentos citados para persuadir os leitores da validade de suas afirmações (GILBERT 1977 apud NICOLAISEN, 2007). No presente artigo, o autor não é uma pessoa, é uma instituição, e que ao emitir uma recomendação certamente tem a intenção de influenciar comportamentos, de modo que as citações passam a ser elementos confirmatórios que auxiliam ao convencimento do público. Como peças de um quebra-cabeça que reúne vontade política e ciência e que faz parte de um processo decisório 
(LOMAS, 2004), as RS citadas, com suas qualidades e deficiências, podem estar cumprindo um papel bem distinto àquele de servir de autêntica prova.

Provavelmente muitas destas recomendações fazem um uso simbólico da citação com o intuito de legitimar um posicionamento, o que estabeleceria uma decisão política a priori (DICKINSON, 2004; DOBBINS et al., 2007a). A citação de uma RS, ao ser identificada $a$ posteriori como respaldo e justificativa, não implica, necessariamente, que os seus achados sejam exclusivos ou parcialmente confirmatórios ou que sua qualidade esteja acima de um determinado limiar. Não obstante, a OMS declarou que segue atentamente as novas investigações e que suas diretrizes se atualizam com RS acompanhadas da avaliação da qualidade dos dados para garantir que estejam baseadas nos melhores dados da pesquisa (WHO, 2011).

Quando a OMS encomendou uma RS sobre a influência da amamentação exclusiva no crescimento e desenvolvimento das crianças (KRAMER; KAKUMA, 2002a), fez um uso instrumental (NUTLEY; WALTER; DAVIES, 2007) desse relatório para mudar a recomendação de amamentação exclusiva de 4 para 6 meses, apesar dos achados não serem conclusivos e que se tenha sinalizado a necessidade de novos maiores e melhores estudos. Nas conclusões foi apontado que não havia evidência objetiva para a questão sob estudo, que era o efeito protetor da amamentação exclusiva contra doenças infecciosas, em contraposição à alegada insuficiência de leite materno exclusivo para satisfazer as necessidades energéticas e de micronutrientes requeridos após o quarto mês de vida. Os autores reportaram que nenhum estudo sugeriu déficit de peso ou estatura em crianças amamentadas exclusivamente até os 6 meses e, por outro lado, que os bebês apresentavam menos morbidade por infecção gastrointestinal e respiratória. Porém o que se observou foi que nessa RS e suas posteriores atualizações (KRAMER; KAKUMA, 2009; 2012), foram selecionados dois únicos ECR, mantidos na sua última versão e categorizados pelos autores como de baixa qualidade e seus dados referem apenas aos aspectos maternos da amamentação. Os estudos observacionais incluídos, que ao longo das atualizações chegaram a ser 23 e categorizados como de qualidade variada, não mudaram as conclusões originais, que permaneceram até a última versão baseadas em três estudos, que tratam das implicações relativas a infecções gastrointestinais e respiratórias em crianças amamentadas. Os estudos que deram origem a esta evidência foram levados a cabo na Bielorrússia, Nigéria e Irã, e, definitivamente, suas evidências são as mais citadas em todas as recomendações.

Por último, ressalta-se que as RO foram difíceis de identificar e de categorizar devido à 
inexistência de uma definição sobre o formato de uma recomendação de saúde, que mostrou ser muito variado. Um aspecto a ser destacado nesse estudo é que a análise das citações permitiu uma reflexão sobre a forma de comunicação entre as instituições que têm um papel norteador para a grande massa de profissionais de saúde. Viu-se que não há um consenso e nem um formato estabelecido, não há uma hierarquia claramente definida, e em princípio, qualquer instituição, associação ou entidade pode emitir seu parecer sobre o tema. Entretanto, a maioria tende a citar e replicar as recomendações da OMS, embora isto não tenha sido especificamente investigado neste estudo.

O pressuposto de que as RO se utilizam das RS como fontes de evidências e de que isto ficaria claro ao analisar as citações não foi confirmado nesta pesquisa. Isto não ocorre porque quatro RS se mostraram frequentemente citadas, enquanto as outras foram pouco ou não citadas.

A importância e o valor de se fazer uma RS ficaram evidenciadas, mas essa prática precisa avançar, sendo rigorosa e relevante, ampliando seu potencial de informar à política e influenciar melhores decisões de saúde da população. Desse modo, recomenda-se que guias e manuais de validade reconhecida sejam consultados para a confecção das RS, e que ferramentas validadas sejam utilizadas para avaliar os estudos a serem incluídos nas RS, para poder serem publicadas e/ou utilizadas como evidências. Por fim, recomenda-se um maior cuidado no relato da pesquisa para que as avaliações e respectivas pontuações reflitam melhor sua qualidade, enquanto as RO precisariam tornar mais transparentes e notórios os processos que levam a instituir e transformar evidências em recomendações.

Finalmente, reconhece-se que esta pesquisa apresenta algumas limitações, especialmente no tocante a que as evidências foram verificadas na seção da discussão e conclusões, com base no relato dos autores, sem uma análise específica sobre os resultados. Por outro lado, quando analisadas as citações nas RO, não foi estudado o momento da citação no texto, mas apenas a existência da citação na lista de referências. E também não foram analisadas as demais citações. Outras análises podem complementar os nossos achados.

\section{REFERÊNCIAS}

AGENCY FOR HEALTHCARE RESEARCH AND QUALITY. Breastfeeding and maternal and infant health outcomes in developed countries. Evidence Report/Technology Assessment No. 153 AHRQ Publication No. 07-E007. Rockville, MD: AHRQ, 2007.

AMERICAN ACADEMY OF PEDIATRICS. Breastfeeding and the use of human milk.

Pediatrics, [s.1.], v. 129, n. 3, p. e827-841, 2012. 
ANDERSON, J. W.; JOHNSTONE, B. M.; REMLEY, D. T. Breast-feeding and cognitive development: a meta-analysis. American Journal of Clinical Nutrition, [s.l.], v. 70, n. 4, p. 525-535, 1999.

ARENZ, S. et al. Breast-feeding and childhood obesity - a systematic review. International Journal of Obesity, [s.1.], v. 28, n. 10, p. 1247-1256, 2004.

BRASIL. Ministério da Saúde. Secretaria de Ciência Tecnologia e Insumos Estratégicos; Departamento de Ciência e Tecnologia. Diretrizes metodológicas: elaboração de revisão sistemática e metanálise de ensaios clínicos randomizados. Brasília: Ministério da Saúde, 2012. (Série A: Normas e manuais técnicos).

BRASIL. MINISTÉRIO DA SAÚDE; DEPARTAMENTO DE CIÊNCIA E TECNOLOGIA. Avaliação de tecnologias em saúde: institucionalização das ações no Ministério da Saúde.

Revista de Saúde Pública, [s.1.], v. 40, n. 4, p. 743-747, 2006.

BRASIL; MINISTÉRIO DA SAÚDE. ORGANIZAÇÃO PAN-AMERICANA DA SAÚDE. Guia alimentar para crianças menores de 2 anos. Brasília: Ministério da Saúde, 2002.

BURDA, B. U.; CHAMBERS, A. R.; JOHNSON, J. C. Appraisal of guidelines developed by the world health organization. Public Health, [s.1.], v. 128, n. 5, p. 444-474, 2014.

CENTRE FOR REVIEWS AND DISSEMINATION. Systematic reviews: CRD's guidance for undertaking reviews in health care. York: University of York, 2009.

DICKINSON, H. D. A sociological perspective on the transfer and utilization of social scientific knowledge for policymaking. In: LIMIEUX-CHARLES, L.; CHAMPAGNE, F. (ed.). Using knowledge and evidence in health care: multidisciplinary perspectives. Toronto: University of Toronto, 2004, p. 41-69.

DOBBINS, M. et al. Public health decision-makers' informational needs and preferences for receiving research evidence. Worldviews on Evidence-Based Nursing, [s.1.], v. 4, n. 3, p. 156-163, 2007a.

FLETCHER, J. What is heterogeneity and is it important? British Medical Journal, [s.1.], v. 334, n. 7584, p. 94-96, 2007.

FRANCO, A.; MALHOTRA, N.; SIMONOVITS, G. Social science. publication bias in the social sciences: Unlocking the file drawer. Science, [s.1.], v. 345, n. 6203, p. 1502-1505, 2014.

GDALEVICH, M. et al. Breast-feeding and the onset of atopic dermatitis in childhood: A systematic review and meta-analysis of prospective studies. Journal of the American Academy of Dermatology, [s.1.], v. 45, n. 4, p. 520-527, 2001.

GREENHALGH, T. Como ler artigos científicos: fundamentos da medicina baseada em evidências. 3. ed. Porto Alegre: Artmed, 2008.

GUYATT, G. et al. A filosofia da medicina baseada em evidências. In: GUYAT, G. et al. Diretrizes para utilização da literatura médica. 2. ed. Porto Alegre: Artmed, 2011a. p. 3137. 
GUYATT, G. et al. Grade guidelines: 1. Introduction-grade evidence profiles and summary of findings tables. Journal of Clinical Epidemiology, [s.1.], v. 64, n. 4, p. 383-394, 2011 b.

HEALTH CANADA et al. Nutrition for healthy term infants: recommendations from birth to six months: A joint statement of Health Canada, Canadian Paediatric Society, Dietitians of Canada and Breastfeeding Committee of Canada. Disponível em: http://www.hc-sc.gc.ca/fnan/nutrition/infant-nourisson/recom/index-eng.php. Acesso em: 18 maio 2018.

HEMINGWAY, P.; BRERETON, N. What is a systematic review? London: Hayward Medical Communication, 2009 (Whatis series).

HIGGINS, J. P. T., et al. Cochrane handbook for systematic reviews of interventions. Version 5.1.0. (updated march 2011). 2011. Disponível em: http://handbook.cochrane.org. Acesso em 15 dez. 2017.

KRAMER, M.; KAKUMA, R. The optimal duration of exclusive breastfeeding: a systematic review. Geneve: WHO, 2002a

KRAMER, M.; KAKUMA, R. Optimal duration of exclusive breastfeeding. The Cochrane Database of Systematic Reviews, n. 1, p. Cd003517, 2002 b.

KRAMER, M.; KAKUMA, R. Optimal duration of exclusive breastfeeding. The Cochrane Database of Systematic Reviews, n. 1, p. CD003517, 2009.

KRAMER, M.; KAKUMA, R. The optimal duration of exclusive breastfeeding: a systematic review. Advances in Experimental Medicine and Biology, v. 554, n., p. 63-77, 2004.

KRAMER, M. S.; KAKUMA, R. Optimal duration of exclusive breastfeeding. The Cochrane Database of Systematic Reviews, n. 8, p. Cd003517, 2012

LAVIS, J. H. et al. How can research organizations more effectively transfer research knowledge to decision makers? The Milbank Quarterly, [s.1.], v. 81, n. 2, p. 221-248, 2003.

LEVINE, M. et al. Dano (estudos observacionais). In: GUYAT, G. et al. Diretrizes para utilização da literatura médica. 2. ed. Porto Alegre: Artmed, 2011, p. 319-334.

LIMIEUX-CHARLES, L.; CHAMPAGNE, F. (eds.). Using knowledge and evidence in health care: multidisciplinary perspectives. Toronto: Univesity of Toronto, 2004

LOHR, K. N. Rating the strength of scientific evidence: Relevance for quality improvement programs. International Journal for Quality in Health Care, [s.1.], v. 16, n. 1, p. 9-18, 2004.

LOMAS, J. et al. Conceptualizing and combining evidence for health system guidance: final report. Ottawa: Canadian Health Service Research Foundation, 2005.

LOMAS, J. Understanding evidence-based decisions-making: or why keyboards are irrational. In: LEMIEUX-CHARLES, L.; CHAMPAGNE, F. (ed.). Using knowledge and 
evidence in health care: multidisciplinary perspectives. Toronto: University of Toronto, 2004. p. 281-290.

MACROBERTS, M. H.; MACROBERTS, B. R. Problems of citation analysis: a study of uncited and seldom-cited influences. Journal of the American Society for Information Science and Technology, [s.1.], v. 61, n. 1, p. 1-13, 2010.

MARTÍNEZ-SILVEIRA, M. S. Bibliotecários são parceiros valiosos em equipes de revisões sistemáticas. CINFORM Encontro Nacional de Ensino e Pesquisa em Informação. 10., 2011. Anais [...] Salvador, Bahia: UFBA 2011.

MEERPOHL, J. J. et al. Scientific value of systematic reviews: survey of editors of core clinical journals. PLoS One, [s.1.], v. 7, n. 5, p. e35732, 2012.

NICOLAISEN, J. Citation analysis. Annual Review of Information Science and Technology, [s.1.], v. 41, n., p. 609-641, 2007.

NUTLEY, S. M.; WALTER, I.; DAVIES, H. T. O. Using evidence: how research can inform public services. Bristol, UK: University of Bristol, 2007

OXMAN, A. D.; LAVIS, J. N.; FRETHEIM, A. Use of evidence in WHO recommendations. Lancet, [s.1.], v. 369, n. 9576, p. 1883-1889, 2007.

PETTICREW, M. et al. Quality-assessed reviews of health care interventions and the database of abstracts of reviews of effectiveness (dare). NHS CRD review, dissemination, and information teams. International Journal of Technology Assessment in Health Care, [s.1.], v. 15 , n. 4, p. 671-678, 1999.

PETTICREW, M.; ROBERTS, H. Systematic reviews in the social sciences: a practical guide. Malden, MA: Blackwell, 2006.

RELEVO, R. Effective search strategies for systematic reviews of medical tests: chapter 4. Journal of General Internal Medicine, [s.1.], v. 27, n. sup. 1, p. S28-32, 2012.

SAMPSON, M. et al. An evidence-based practice guideline for the peer review of electronic search strategies. Journal of Clinical Epidemioly, [s.1.], v. 62, n. 9, p. 944-952, 2009.

SHEA, B. J. et al. AMSTAR is a reliable and valid measurement tool to assess the methodological quality of systematic reviews. Journal of Clinical Epidemiology, [s.l.], v. 62, n. 10, p. 1013-1020, 2009.

SHEA, B. J. et al. External validation of a measurement tool to assess systematic reviews (AMSTAR). PLoS One, [s.1.], v. 2, n. 12, p. e1350, 2007.

TITLER, M. G. The evidence for evidence-based practice implementation. In: HUGHES, R. G. (ed.). Patient safety and quality: an evidence-based handbook for nurses. Rockville, MD: AHRQ, 2008. cap. 7, p. 1-49

UPSHUR, R. E. G. Seven characteristics of medical evidence. Journal of Evaluation in Clinical Practice, [s.1.], v. 6, n. 2, p. 93-97, 2000. 
WORLD HEALTH ORGANIZATION COLLABORATIVE STUDY TEAM ON THE ROLE OF BREASTFEEDING ON THE PREVENTION OF INFANT MORTALITY. Effect of breastfeeding on infant and child mortality due to infectious diseases in less developed countries: a pooled analysis. Lancet, v. 355, n. 9202, p. 451-455, 2000.

WORLD HEALTH ORGANIZATION. Planning guide for national implementation of the global strategy for infant and young child feeding. Geneva: WHO, 2007.

WORLD HEALTH ORGANIZATION. WHO handbook for guideline development. Geneva: WHO, 2012.

WORLD HEALTH ORGANIZATION. Exclusive breastfeeding for six months best for babies everywhere. 2011. Disponível em:

http://www.who.int/mediacentre/news/statements/2011/breastfeeding_20110115/en/\%3E. Acesso em: 10 jan. 2019.

WORLD HEALTH ORGANIZATION. Infant and young child feeding: model chapter for textbooks for medical students and allied health professional. Geneva: WHO, 2010. 


\section{APENDICE A}

Tabela 1 - Características das Revisões sistemáticas citadas

\begin{tabular}{|c|c|c|c|c|c|c|c|c|c|}
\hline 竞 & $\frac{\stackrel{0}{\frac{0}{0}}}{\frac{0}{0}}$ & 气̆ & 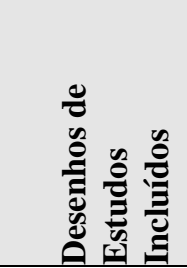 & 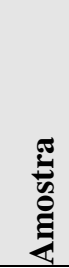 & 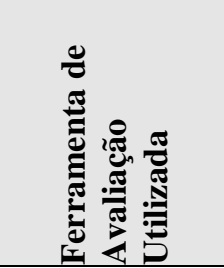 & $\Sigma$ & 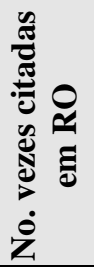 & 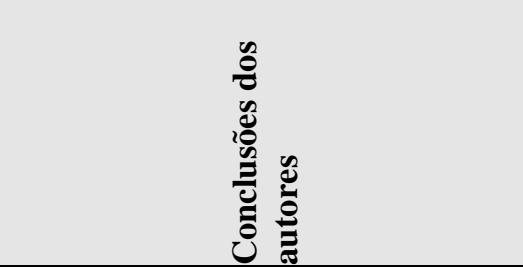 & 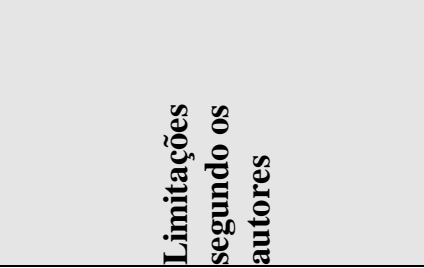 \\
\hline $\begin{array}{l}\text { Akobeng } \\
2006\end{array}$ & $\begin{array}{l}\text { Efeito da AM no } \\
\text { risco de doença } \\
\text { celíaca }\end{array}$ & UK & $6 \mathrm{CC}$ & 8 & $\begin{array}{l}\text { Critical } \\
\text { Appraisal } \\
\text { Skills } \\
\text { Program }\end{array}$ & $\mathrm{S}$ & 2 & $\begin{array}{l}\text { Aumentar o tempo de AM reduz } \\
\text { significativamente o risco de DC. } \\
\text { Não está claro se AM só retarda o } \\
\text { aparecimento dos sintomas ou se dá } \\
\text { proteção permanente contra a } \\
\text { doença. }\end{array}$ & $\begin{array}{l}\text { Necessário um estudo de } \\
\text { coorte prospectivo de longo } \\
\text { prazo. Falhas na } \\
\text { classificação de AM e ajuste } \\
\text { de fatores de confundimento. }\end{array}$ \\
\hline $\begin{array}{l}\text { Anderson } \\
1999\end{array}$ & $\begin{array}{l}\text { AM e } \\
\text { desenvolvimento } \\
\text { cognitivo }\end{array}$ & USA & $20 \mathrm{C}$ & 3 & $\mathrm{~N}$ & $S$ & 12 & $\begin{array}{l}\text { Crianças AM tem um } \\
\text { desenvolvimento cognitivo } 3.16 \\
\text { pontos maior que os AF. Diferença } \\
\text { observada desde os } 6 \text { meses até } 15 \\
\text { anos. Quanto mais tempo AM } \\
\text { maior diferenças no } \\
\text { desenvolvimento cognitivo. Os } \\
\text { BPN tem mais benefícios. }\end{array}$ & $\begin{array}{l}\text { Diferenças entre fatores de } \\
\text { confundimento não } \\
\text { mensuradas ou mal avaliadas } \\
\text { podem modificar o } \\
\text { resultado. Necessários mais } \\
\text { estudos para responder esta } \\
\text { questão. }\end{array}$ \\
\hline $\begin{array}{l}\text { Arenz } \\
2004\end{array}$ & $\begin{array}{l}\text { AM e obesidade } \\
\text { infantil }\end{array}$ & Alemanha & $\begin{array}{l}15 \mathrm{C} ; 2 \mathrm{CC} ; \\
11 \mathrm{CS}\end{array}$ & 7 & $\mathrm{~N}$ & $\mathrm{~S}$ & 8 & $\begin{array}{l}\text { AM associada a um pequeno porém } \\
\text { consistente efeito protetor contra } \\
\text { obesidade na criança. }\end{array}$ & $\begin{array}{l}\text { Viés de publicação e número } \\
\text { pequeno de estudos }\end{array}$ \\
\hline $\begin{array}{l}\text { Bachrach } \\
2003\end{array}$ & $\begin{array}{l}\text { AM e risco de } \\
\text { hospitalização por } \\
\text { doença } \\
\text { respiratória na } \\
\text { infância }\end{array}$ & USA & $\begin{array}{l}7 \mathrm{C} ; 1 \mathrm{CS} ; \\
1 \mathrm{OUT}\end{array}$ & 6 & $\mathrm{~N}$ & $\mathrm{~S}$ & 5 & $\begin{array}{l}\text { Crianças AF são associadas a um } \\
\text { aumento } 3.6 \text { vezes do risco de } \\
\text { hospitalização por DR se } \\
\text { comparadas a crianças } \\
\text { exclusivamente AM por } 4 \text { meses }\end{array}$ & $\begin{array}{l}\text { Somente com novos e } \\
\text { melhores estudos que } \\
\text { quantifiquem } \\
\text { apropriadamente dados sobre } \\
\text { AM se terão dados } \\
\text { suficientes para ajudar na } \\
\text { escolha de amamentar. }\end{array}$ \\
\hline $\begin{array}{l}\text { Barclay } \\
2009\end{array}$ & $\begin{array}{l}\text { AM e doença } \\
\text { inflamatória } \\
\text { intestinal } \\
\text { pediátrica }\end{array}$ & UK & $8 \mathrm{CC}$ & 7 & $\begin{array}{l}\text { Scottish } \\
\text { Intercollegiate } \\
\text { Guideline } \\
\text { network }\end{array}$ & $\mathrm{S}$ & 1 & $\begin{array}{l}\text { Possível efeito protetor da AM para } \\
\text { o aparecimento precoce de doença } \\
\text { inflamatória intestinal. }\end{array}$ & $\begin{array}{l}\text { A qualidade dos dados é } \\
\text { pobre, Os achados devem ser } \\
\text { investigados em melhores } \\
\text { estudos. }\end{array}$ \\
\hline
\end{tabular}

P2P \& INOVAÇÃO, Rio de Janeiro, v. 5 n. 2, p.133-174, Mar./Ago. 2019. 


\begin{tabular}{|c|c|c|c|c|c|c|c|c|c|}
\hline 冚 & $\frac{0}{0}$ & 气ี & 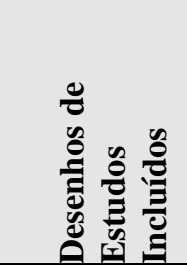 & 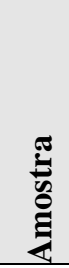 & 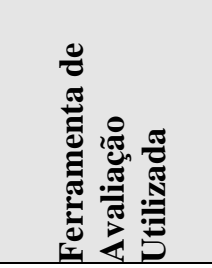 & $\sum$ & 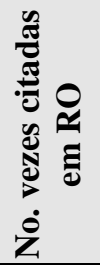 & 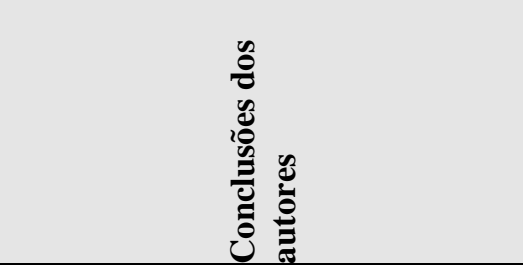 & 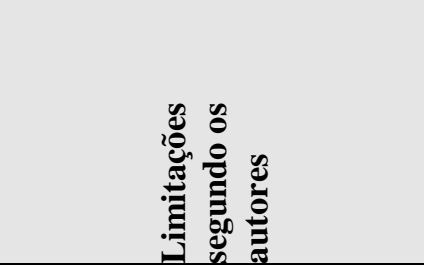 \\
\hline $\begin{array}{l}\text { Der } \\
2006\end{array}$ & $\begin{array}{l}\text { Efeitos da AM na } \\
\text { inteligência da } \\
\text { criança }\end{array}$ & UK & $\begin{array}{l}9 \mathrm{CC} ; \mathrm{OUT} \\
\mathrm{NI} \mathrm{a} \\
\text { quantidade }\end{array}$ & 0 & $\mathrm{~N}$ & $S$ & 2 & $\begin{array}{l}\text { AM tem um pequeno ou nenhum } \\
\text { efeito na inteligência da criança. }\end{array}$ & $\begin{array}{l}\text { A associação observada } \\
\text { entre AM e desenvolvimento } \\
\text { cognitivo é resultado do } \\
\text { fator confundidor: } \\
\text { inteligência da mãe. }\end{array}$ \\
\hline $\begin{array}{l}\text { Gdalevich } \\
\text { 2001a }\end{array}$ & $\begin{array}{l}\text { AM e o } \\
\text { aparecimento de } \\
\text { dermatite atópica }\end{array}$ & Israel & $10 \mathrm{C}$ & 6 & $\begin{array}{l}\text { Critérios de } \\
\text { Kramer, } 1988\end{array}$ & $S$ & 4 & $\begin{array}{l}\text { Efeito protetor significativo da AM } \\
\text { exclusiva por } 3 \text { meses ou mais na } \\
\text { prevenção de DA na criança. Maior } \\
\text { efeito em crianças de famílias com } \\
\text { atopias. O efeito é menor na } \\
\text { população em geral e não existe em } \\
\text { crianças sem parentes de primeira } \\
\text { ordem atópicos. }\end{array}$ & $\begin{array}{l}\text { Não pode excluir } \\
\text { possibilidade de viés de } \\
\text { memória, falhas na definição } \\
\text { dos critérios diagnósticos, } \\
\text { diferentes tempos de } \\
\text { seguimento, veracidade das } \\
\text { declarações dos } \\
\text { entrevistados e intervenção } \\
\text { de fatores de confundimento. }\end{array}$ \\
\hline $\begin{array}{l}\text { Gdalevich } \\
\text { 2001b }\end{array}$ & $\begin{array}{l}\text { AM e risco de } \\
\text { asma em crianças }\end{array}$ & Israel & $12 \mathrm{C}$ & 6 & $\begin{array}{l}\text { Critérios de } \\
\text { Kramer, } 1988\end{array}$ & $\mathrm{~S}$ & 4 & $\begin{array}{l}\text { AM exclusiva durante o } 1^{\circ} \text { mês está } \\
\text { associada às menores taxas de asma } \\
\text { na infância. Efeito protetor } \\
\text { substancial da AM em crianças } \\
\text { com história de atopias. }\end{array}$ & $\begin{array}{l}\text { Não pode excluir } \\
\text { possibilidade de viés nos } \\
\text { estudos originais. Não foi } \\
\text { possível o ajuste de fatores } \\
\text { de confundimento. } \\
\text { Estudos apenas em países } \\
\text { desenvolvidos. }\end{array}$ \\
\hline $\begin{array}{l}\text { Harder } \\
2005\end{array}$ & $\begin{array}{l}\text { Duração da AM e } \\
\text { risco de obesidade } \\
\text { adulta }\end{array}$ & Alemanha & $16 \mathrm{C} ; 1 \mathrm{CC}$ & 5 & $\mathrm{~N}$ & $\mathrm{~S}$ & 4 & $\begin{array}{l}\text { A duração da AM é inversa e } \\
\text { linearmente associada ao risco de } \\
\text { sobrepeso. O prolongamento da } \\
\text { AM mesmo parcial por até } 9 \text { meses } \\
\text { leva a uma diminuição do risco de } \\
\text { sobrepeso na vida tardia. } 4 \% \text { por } \\
\text { cada mês de AM. Apesar de } \\
\text { pequena a associação é causal. O } \\
\text { mecanismo pelo qual AM afeta o } \\
\text { risco de sobrepeso não está ainda } \\
\text { esclarecido. }\end{array}$ & $\begin{array}{l}\text { Tamanho da amostra } \\
\text { pequeno. Tipo e número de } \\
\text { fatores de confundimento } \\
\text { difere entre estudos. Os } \\
\text { estudos são análise } \\
\text { secundária de inquéritos de } \\
\text { saúde concebidos para } \\
\text { responder outras questões. }\end{array}$ \\
\hline
\end{tabular}

P2P \& INOVAÇÃO, Rio de Janeiro, v. 5 n. 2, p.133-174, Mar./Ago. 2019. 


\begin{tabular}{|c|c|c|c|c|c|c|c|c|c|}
\hline : & $\frac{0}{\stackrel{0}{\frac{0}{0}}}$ & 气 & 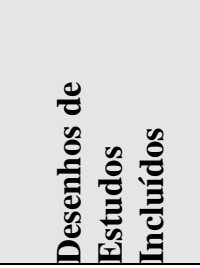 & $\stackrel{5}{\stackrel{5}{5}}$ & 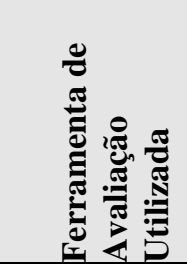 & $\Sigma$ & 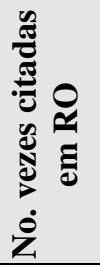 & 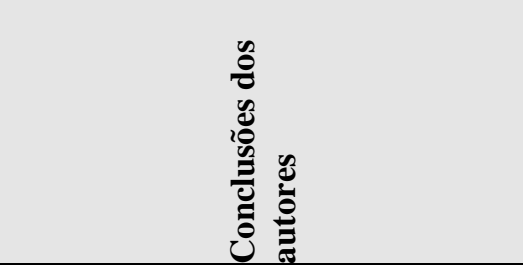 & 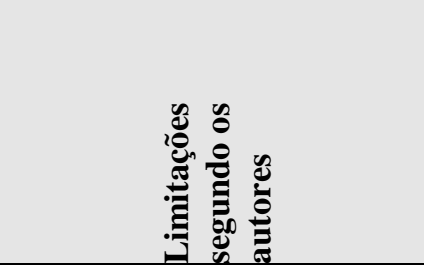 \\
\hline $\begin{array}{l}\text { Hauck } \\
2011\end{array}$ & $\begin{array}{l}\text { AM e redução do } \\
\text { risco de morte } \\
\text { súbita infantil }\end{array}$ & USA & $18 \mathrm{CC}$ & 4 & $\mathrm{~N}$ & $\mathrm{~S}$ & 3 & $\begin{array}{l}\text { AM de qualquer duração protege } \\
\text { contra MSL e é mais forte se for } \\
\text { exclusiva. }\end{array}$ & $\begin{array}{l}\text { Faltam estudos com dados } \\
\text { sobre a duração da AM e } \\
\text { quando presentes há } \\
\text { diferentes formas de medir a } \\
\text { duração. Possível viés de } \\
\text { publicação. }\end{array}$ \\
\hline $\begin{array}{l}\text { Henderson } \\
2001\end{array}$ & $\begin{array}{l}\text { Fórmula vs. leite } \\
\text { materno de termo } \\
\text { no crescimento do } \\
\text { pré-termo ou } \\
\text { baixo-peso ao } \\
\text { nascer }\end{array}$ & Austrália & $6 \mathrm{RCT}$ & 8 & $\begin{array}{l}\text { Critérios } \\
\text { Cochrane }\end{array}$ & $\mathrm{S}$ & 1 & $\begin{array}{l}\text { Em PT e BPN uso de AF vs leite } \\
\text { humano de termo não fortificado } \\
\text { leva a taxas maiores de crescimento } \\
\text { no curto prazo. }\end{array}$ & $\begin{array}{l}\text { Dados limitados, não } \\
\text { conclusões definitivas sobre } \\
\text { efeitos adversos da AF. Não } \\
\text { há RCT comparando AF e } \\
\text { leite humana fortificada }\end{array}$ \\
\hline $\begin{array}{l}\text { Horta } \\
2007\end{array}$ & $\begin{array}{l}\text { Efeitos de longo } \\
\text { prazo da AM } * *\end{array}$ & Brasil & $\begin{array}{l}2 \mathrm{RCT} ; 17 \mathrm{C} \\
11 \mathrm{CS}\end{array}$ & 6 & $\begin{array}{l}\text { Critérios do } \\
\text { autor }\end{array}$ & $\mathrm{S}$ & 12 & $\begin{array}{l}\text { AM tem efeitos benéficos ao longo } \\
\text { prazo. } \\
\text { Hipertensão = efeito pequeno, } \\
\text { porém significativo; efeito protetor } \\
\text { Colesterol= Não tem efeito } \\
\text { significativo em crianças e } \\
\text { adolescentes, efeito pequeno em } \\
\text { adultos } \\
\text { Obesidade = efeito protetor } \\
\text { pequeno na prevalência } \\
\text { DMT2 = efeito possível } \\
\text { Inteligência= pouco aumento }\end{array}$ & $\begin{array}{l}\text { Efeitos estatisticamente } \\
\text { significantes, porém as vezes } \\
\text { modestos. Nenhum estudo } \\
\text { atingiu níveis de confiança } \\
\text { perfeitos. Precisaria um } \\
\text { estudo de coorte de longo } \\
\text { prazo. }\end{array}$ \\
\hline $\begin{array}{l}\text { Klement } \\
2004\end{array}$ & $\begin{array}{l}\text { AM e risco de } \\
\text { doença } \\
\text { inflamatória } \\
\text { intestinal }\end{array}$ & Israel & $\begin{array}{l}15 \mathrm{CC} ; 2 \\
\text { OUT }\end{array}$ & 7 & $\begin{array}{l}\text { Critérios do } \\
\text { autor }\end{array}$ & $\mathrm{S}$ & 2 & $\begin{array}{l}\text { Resultados suporta a hipótese de } \\
\text { associação entre AM e baixo risco } \\
\text { de doença de Crohn e colite } \\
\text { ulcerativa }\end{array}$ & $\begin{array}{l}\text { Somente poucos estudos de } \\
\text { qualidade. Precisam-se mais } \\
\text { estudos para reforçar estas } \\
\text { observações. Grande } \\
\text { heterogeneidade e viés de } \\
\text { publicação foi detectado }\end{array}$ \\
\hline
\end{tabular}




\begin{tabular}{|c|c|c|c|c|c|c|c|c|c|}
\hline 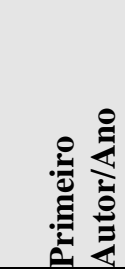 & $\frac{\stackrel{2}{0}}{\frac{2}{0}}$ & 气̆ & 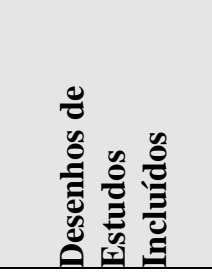 & 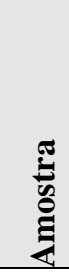 & 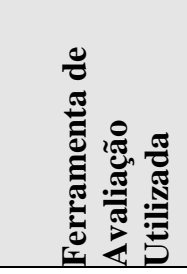 & $\Sigma$ & 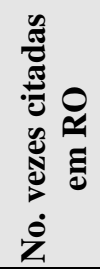 & 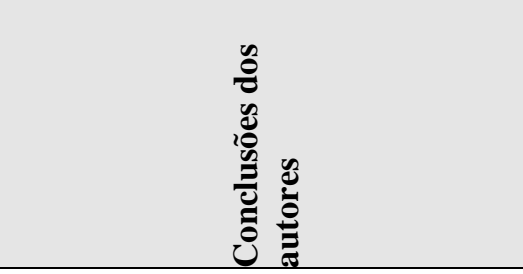 & 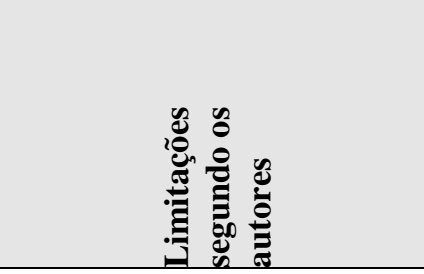 \\
\hline $\begin{array}{l}\text { Kramer } \\
2002 a\end{array}$ & $\begin{array}{l}\text { Duração da AM } \\
\text { exclusiva e saúde } \\
\text { da criança, } \\
\text { crescimento e } \\
\text { desenvolvimento } \\
\text { (Publicada pela } \\
\text { WHO) }\end{array}$ & Canadá & $\begin{array}{l}2 \mathrm{RCT} ; 13 \mathrm{C} ; \\
1 \mathrm{CS}\end{array}$ & 7 & $\begin{array}{l}\text { Critérios } \\
\text { Cochrane }\end{array}$ & $\mathrm{S}$ & 15 & $\begin{array}{l}\text { Não há risco de carência nutricional } \\
\text { ao recomendar a AM exclusiva por } \\
6 \text { messes como política em países } \\
\text { desenvolvidos e em } \\
\text { desenvolvimento. Crianças tem } \\
\text { menos morbidades por infecções } \\
\text { gastrointestinais e não foi } \\
\text { observado déficit do crescimento. } \\
\text { AM exclusiva não modifica os } \\
\text { riscos dos bebês apresentarem } \\
\text { outras infecções, doenças alérgicas, } \\
\text { obesidade, cáries dentárias ou } \\
\text { problemas cognitivos ou } \\
\text { comportamentais até a idade } \\
\text { escolar. Em países em } \\
\text { desenvolvimento, AM exclusiva } \\
\text { pode levar a uma redução do nível } \\
\text { de ferro dos bebês. }\end{array}$ & $\begin{array}{l}\text { Incertezas remanescentes } \\
\text { devem ser resolvidas com } \\
\text { RCT. Variação considerável } \\
\text { da definição de AM entre os } \\
\text { estudos. } \\
\text { Estudos controlados de baixa } \\
\text { qualidade porém superior a } \\
\text { qualquer observacional. }\end{array}$ \\
\hline $\begin{array}{l}\text { Kramer } \\
2002 b\end{array}$ & $\begin{array}{l}\text { Idem } \\
\text { (Publicada pela } \\
\text { Cochrane) }\end{array}$ & Idem & $\begin{array}{l}2 \mathrm{RCT} ; 18 \mathrm{C} ; \\
2 \mathrm{CS}\end{array}$ & & $\begin{array}{l}\text { Critérios } \\
\text { Cochrane }\end{array}$ & $\mathrm{S}$ & 3 & & \\
\hline $\begin{array}{l}\text { Kramer } \\
* * * \\
2004\end{array}$ & $\begin{array}{l}\text { Idem } \\
\text { (Estudo duplicado } \\
\text { publicado em } \\
\text { periódico) }\end{array}$ & Idem & Idem & & Idem & $\mathrm{S}$ & 2 & & \\
\hline $\begin{array}{l}\text { Kramer } \\
2009\end{array}$ & $\begin{array}{l}\text { Idem } \\
\text { (Atualização } \\
\text { Cochrane 2007) }\end{array}$ & Idem & $\begin{array}{l}2 \mathrm{RCT} ; 18 \mathrm{C} ; \\
2 \mathrm{CS}\end{array}$ & & Idem & $\mathrm{S}$ & 3 & & \\
\hline
\end{tabular}




\begin{tabular}{|c|c|c|c|c|c|c|c|c|c|}
\hline : & $\frac{\substack{0 \\
\frac{0}{0}}}{0}$ & 气̆ & 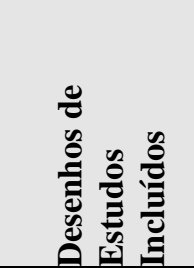 & 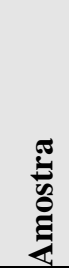 & 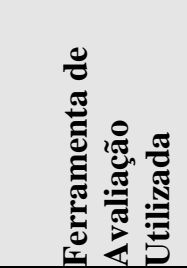 & $\Sigma$ & 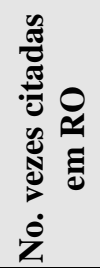 & 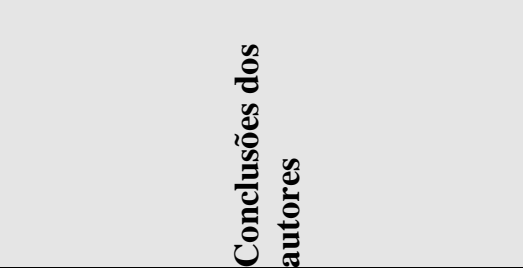 & 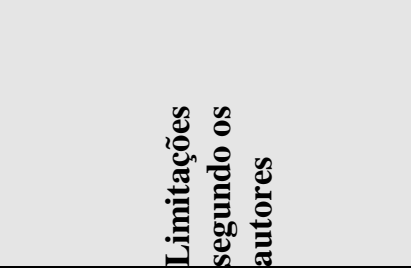 \\
\hline $\begin{array}{l}\text { Kwan } \\
2004\end{array}$ & $\begin{array}{l}\text { AM e risco de } \\
\text { leucemia infantil }\end{array}$ & USA & $14 \mathrm{CC}$ & 6 & $\mathrm{~N}$ & $\mathrm{~S}$ & 4 & $\begin{array}{l}\text { AM de curto e de longo prazo tem } \\
\text { potencial efeito protetor do risco de } \\
\text { leucemia linfoblástica e efeito } \\
\text { possível na leucemia mieloide. } \\
\text { O efeito porém, é visto com } \\
\text { ressalvas. }\end{array}$ & $\begin{array}{l}\text { Problemas com os desenhos } \\
\text { dos estudos e a classificação } \\
\text { da doença. }\end{array}$ \\
\hline $\begin{array}{l}\text { Martin } \\
2005\end{array}$ & $\begin{array}{l}\text { AM e hipertensão } \\
\text { adulta }\end{array}$ & UK & $\begin{array}{l}2 \mathrm{RCT} ; 9 \mathrm{C} ; \\
4 \mathrm{CS}\end{array}$ & 6 & $\mathrm{~N}$ & $\mathrm{~S}$ & 2 & $\begin{array}{l}\text { Uma pequena redução da PA } \\
\text { diastólica foi associada com AM } \\
\text { independentemente do tamanho do } \\
\text { estudo. Os estudos pequenos } \\
\text { reportam maiores diferenças na } \\
\text { média da PA sistólica entre os AM, } \\
\text { porém os estudos grandes reportam } \\
\text { menor diferença. }\end{array}$ & $\begin{array}{l}\text { Evidências de } \\
\text { heterogeneidade entre os } \\
\text { estudos. } \\
\text { Poucos estudos controlaram } \\
\text { fatores de confundimento. }\end{array}$ \\
\hline $\begin{array}{l}\text { Martin } \\
2004\end{array}$ & $\begin{array}{l}\text { AM e mortalidade } \\
\text { cardiovascular }\end{array}$ & UK & $4 \mathrm{C}$ & 6 & $\begin{array}{l}\text { Critérios do } \\
\text { autor }\end{array}$ & $\mathrm{S}$ & 1 & $\begin{array}{l}\text { Evidência pequena, mas consistente } \\
\text { da influência da AM nos desfechos } \\
\text { subsequentes a todo tipo de causa } \\
\text { de mortalidade por DCV. AM não } \\
\text { está associado a mortalidade por } \\
\text { DCV, mas há possibilidades de que } \\
\text { AM prolongada esteja associada a } \\
\text { um aumento de } 16 \% \text { de mortes por } \\
\text { DCV }\end{array}$ & $\begin{array}{l}\text { Há viés de publicação e } \\
\text { fatores de confundimento } \\
\text { residuais }\end{array}$ \\
\hline $\begin{array}{l}\text { McGuire, } \\
2003\end{array}$ & $\begin{array}{l}\text { Leite humana } \\
\text { doada } v s \text { fórmula } \\
\text { na prevenção de } \\
\text { enterocolite em } \\
\text { pré-termo }\end{array}$ & Escócia & $4 \mathrm{RCT}$ & 6 & $\begin{array}{l}\text { Critérios } \\
\text { Cochrane }\end{array}$ & $\mathrm{S}$ & 1 & $\begin{array}{l}\text { Alimentação com leite humano } \\
\text { doado está associada a um risco } \\
\text { relativo reduzido de EN. Crianças } \\
\text { PT e BPN alimentadas com leite } \\
\text { humano têm } 3 \text { vezes menos } \\
\text { chances de desenvolver EN e } 4 \\
\text { vezes menos chances de serem } \\
\text { confirmadas com EN. }\end{array}$ & $\begin{array}{l}\text { Os dados são antigos e estes } \\
\text { achados não são aplicáveis à } \\
\text { moderna população de PT e } \\
\text { BPN. Não há aplicabilidade } \\
\text { clinica dos achados }\end{array}$ \\
\hline
\end{tabular}

P2P \& INOVAÇÃO, Rio de Janeiro, v. 5 n. 2, p.133-174, Mar./Ago. 2019. 


\begin{tabular}{|c|c|c|c|c|c|c|c|c|c|}
\hline 象这 & $\frac{\substack{0 \\
\frac{0}{0}}}{0}$ & 气̆ & 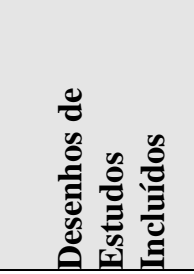 & 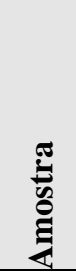 & 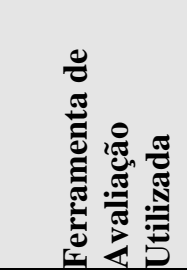 & $\Sigma$ & 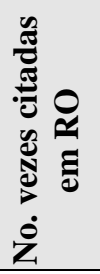 & 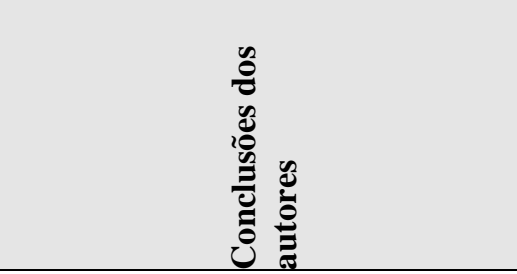 & 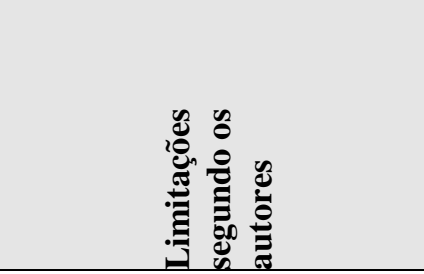 \\
\hline $\begin{array}{l}\text { Norris } \\
1996\end{array}$ & $\begin{array}{l}\text { AM e risco de } \\
\text { diabetes Tipo } 1\end{array}$ & USA & $17 \mathrm{CC}$ & 6 & $\begin{array}{l}\text { Critérios do } \\
\text { autor }\end{array}$ & $\mathrm{N}$ & 2 & $\begin{array}{l}\text { O aumento do risco de DMT1 } \\
\text { associado a AM é pequeno. A fraca } \\
\text { associação entre dieta infantil e } \\
\text { DMT1 pode ter explicações nas } \\
\text { falhas metodológicas }\end{array}$ & $\begin{array}{l}\text { Muito viés e distorções nos } \\
\text { grupos casos e controles }\end{array}$ \\
\hline $\begin{array}{l}\text { Owen } \\
2005\end{array}$ & $\begin{array}{l}\text { AM e risco de } \\
\text { obesidade ao } \\
\text { longo da vida }\end{array}$ & UK & $\begin{array}{l}17 \mathrm{C} ; 1 \mathrm{CC} ; \\
10 \mathrm{CS}\end{array}$ & 6 & $\mathrm{~N}$ & $\mathrm{~S}$ & 4 & $\begin{array}{l}\text { AM protege contra obesidade na } \\
\text { vida adulta. AM inicial está } \\
\text { associada a um risco reduzido de } \\
\text { obesidade comparada à AF. } \\
\text { Evidência forte em estudos } \\
\text { pequenos que ainda permanece em } \\
\text { estudos maiores. Nos estudos que } \\
\text { ajustaram para os fatores de } \\
\text { confusão houve redução expressiva } \\
\text { da associação. }\end{array}$ & $\begin{array}{l}\text { A magnitude da associação } \\
\text { permanece sem ser } \\
\text { esclarecida. Necessários } \\
\text { dados sistemáticos dos } \\
\text { efeitos da duração de AM na } \\
\text { obesidade. Novos estudos } \\
\text { preferencialmente } \\
\text { controlados e randomizados } \\
\text { que examinem os efeitos dos } \\
\text { fatores de confundimento e } \\
\text { do viés de publicação }\end{array}$ \\
\hline $\begin{array}{l}\text { Owen } \\
2002\end{array}$ & $\begin{array}{l}\text { AM e colesterol } \\
\text { no sangue na } \\
\text { infância e } \\
\text { adolescência. }\end{array}$ & UK & $26 \mathrm{C} ; 13 \mathrm{CS}$ & 5 & $\mathrm{~N}$ & $\mathrm{~S}$ & 2 & $\begin{array}{l}\text { A média de CT em crianças e } \\
\text { adolescentes não está relacionada } \\
\text { com padrões de alimentação na } \\
\text { infância. Na infância a média do } \\
\text { CT foi maior entre AM e nos } \\
\text { adultos essa média foi menor entre } \\
\text { os AM. AM está associada ao } \\
\text { aumento da média de CT e LDL na } \\
\text { infância e baixos níveis na vida } \\
\text { adulta. AM tem benefícios no } \\
\text { longo prazo para a saúde } \\
\text { cardiovascular. }\end{array}$ & $\begin{array}{l}\text { Não se sabe a duração da } \\
\text { AM requerida para a redução } \\
\text { de CT no adulto. A } \\
\text { causalidade ainda precisa ser } \\
\text { demonstrada. }\end{array}$ \\
\hline
\end{tabular}




\begin{tabular}{|c|c|c|c|c|c|c|c|c|c|}
\hline 竞 & $\frac{\stackrel{2}{a}}{\frac{0}{0}}$ & 气 & 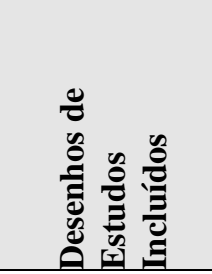 & 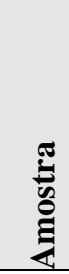 & 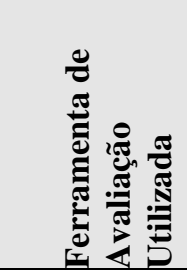 & $\sum$ & 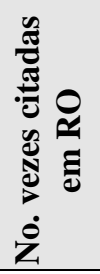 & 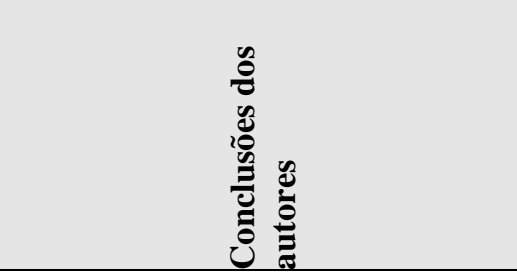 & 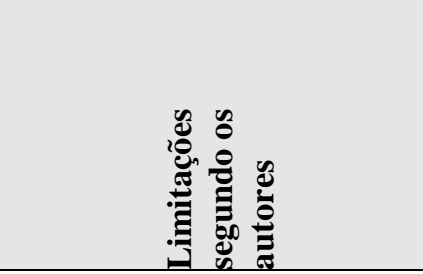 \\
\hline $\begin{array}{l}\text { Owen } \\
2003\end{array}$ & $\begin{array}{l}\text { Efeitos da AM na } \\
\text { hipertensão adulta }\end{array}$ & UK & $\begin{array}{l}1 \mathrm{RCT} .11 \mathrm{C} \text {; } \\
12 \mathrm{CS}\end{array}$ & 3 & $\begin{array}{l}\text { Critérios do } \\
\text { Autor }\end{array}$ & $\mathrm{S}$ & 1 & $\begin{array}{l}\text { AM tem pelo menos um modesto } \\
\text { efeito protetor da PA. }\end{array}$ & $\begin{array}{l}\text { Uma seleção de estudos } \\
\text { positivos e pequenos pode } \\
\text { ter exagerado a afirmação de } \\
\text { que AM reduz a pressão } \\
\text { sistólica na vida tardia. O } \\
\text { efeito modesto demonstrado } \\
\text { nos estudos grandes não tem } \\
\text { importância clínica ou de } \\
\text { saúde publica }\end{array}$ \\
\hline $\begin{array}{l}\text { Owen } \\
2006\end{array}$ & $\begin{array}{l}\text { AM e sua } \\
\text { influência no risco } \\
\text { de diabetes Tipo } 2 \\
\text { no adulto }\end{array}$ & UK & $\begin{array}{l}1 \mathrm{RCT} ; 9 \mathrm{C} ; \\
1 \mathrm{CC}, 12 \mathrm{CS}\end{array}$ & 6 & $\mathrm{~N}$ & $\mathrm{~S}$ & 3 & $\begin{array}{l}\text { AM associada a risco reduzido de } \\
\text { DMT2 quando comparada à AF. } \\
\text { Crianças e adultos que foram AM } \\
\text { têm valores marginais de } \\
\text { concentração de insulina mais } \\
\text { baixos do AF. Crianças AM tem } \\
\text { menor nível de glucose pós- } \\
\text { prandial no sangue que os AF. }\end{array}$ & $\begin{array}{l}\text { Ainda é preciso saber se AM } \\
\text { protege contra DMT2, a } \\
\text { extensão dessa proteção e a } \\
\text { duração da AM necessária } \\
\text { para proteção. }\end{array}$ \\
\hline $\begin{array}{l}\text { Quigley } \\
2007\end{array}$ & $\begin{array}{l}\text { Fórmula } v s \text {. leite } \\
\text { materno doado no } \\
\text { crescimento do } \\
\text { pré-termo ou } \\
\text { baixo-peso ao } \\
\text { nascer }\end{array}$ & UK & $8 \mathrm{RCT}$ & 8 & $\begin{array}{l}\text { Critérios } \\
\text { Cochrane }\end{array}$ & $\mathrm{S}$ & 2 & $\begin{array}{l}\text { PT e BPN AF vs leite humano } \\
\text { doado resultam em taxa maior de } \\
\text { crescimento em menor prazo e } \\
\text { também maior risco de desenvolver } \\
\text { enterocolite necrosante. }\end{array}$ & $\begin{array}{l}\text { Dados limitados para } \\
\text { comparação de AF vs leite } \\
\text { materno doado fortificado } \\
\text { com nutrientes. Limitação } \\
\text { para indicação e aplicação de } \\
\text { achados. }\end{array}$ \\
\hline $\begin{array}{l}\text { Shah } \\
2006\end{array}$ & $\begin{array}{l}\text { AM ou leite } \\
\text { humano e alívio } \\
\text { nos procedimentos } \\
\text { dolorosos no } \\
\text { neonato } \\
\text { (Publicação da } \\
\text { Cochrane) }\end{array}$ & Canadá & $11 \mathrm{RCT}$ & 8 & $\begin{array}{l}\text { Critérios } \\
\text { Cochrane }\end{array}$ & $\mathrm{S}$ & 1 & $\begin{array}{l}\text { Se disponível a AM deve ser usada } \\
\text { para aliviar procedimentos } \\
\text { dolorosos em neonatos comparado } \\
\text { ao placebo, à postura ou a não } \\
\text { intervenção. Administrar glucose } \\
\text { produz efeito similar à AM. A } \\
\text { efetividade de AM para } \\
\text { procedimentos repetidos dolorosos } \\
\text { não foi estabelecida. }\end{array}$ & $\begin{array}{l}\text { Muita heterogeneidade no } \\
\text { controle da intervenção e na } \\
\text { avaliação da dor nos estudos } \\
\text { originais. }\end{array}$ \\
\hline
\end{tabular}

P2P \& INOVAÇÃO, Rio de Janeiro, v. 5 n. 2, p.133-174, Mar./Ago. 2019. 


\begin{tabular}{|c|c|c|c|c|c|c|c|c|c|}
\hline 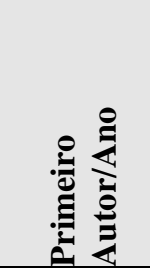 & $\frac{0}{\frac{2}{0}}$ & 气 & 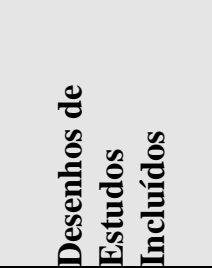 & 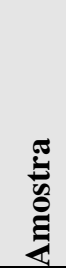 & 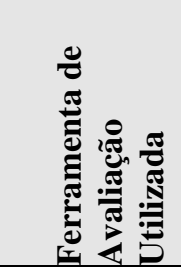 & $\Sigma$ & 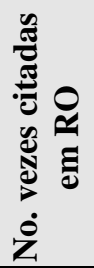 & 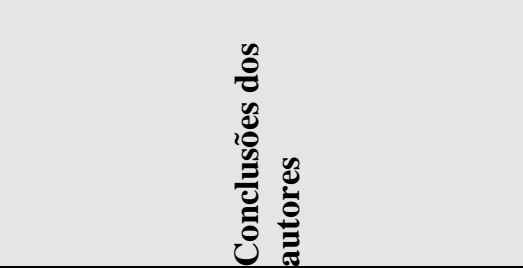 & 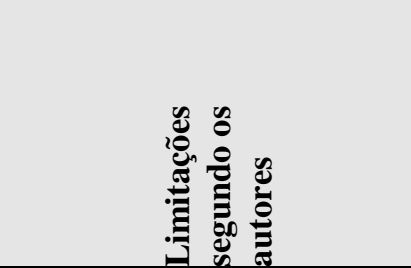 \\
\hline $\begin{array}{l}\text { Shah *** } \\
2007\end{array}$ & $\begin{array}{l}\text { Idem } \\
\text { (Estudo duplicado } \\
\text { publicado em } \\
\text { periódico) }\end{array}$ & Idem & Idem & & Idem & $\mathrm{S}$ & 1 & & \\
\hline $\begin{array}{l}\text { Valaitis } \\
2000\end{array}$ & $\begin{array}{l}\text { A relação entre } \\
\text { AM e caries } \\
\text { infantil }\end{array}$ & Canadá & $\begin{array}{l}24 \mathrm{CC} ; 1 \mathrm{CS} ; \\
3 \mathrm{OUT}\end{array}$ & 3 & $\begin{array}{l}\text { Critérios } \\
\text { Cochrane }\end{array}$ & $\mathrm{N}$ & 2 & $\begin{array}{l}\text { Estudos de qualidade moderada } \\
\text { indicam que AM durante1 ano e à } \\
\text { noite após a erupção dos dentes, } \\
\text { pode estar associada à cáries na } \\
\text { infância. Não se pode definir o } \\
\text { momento em que a criança deva ser } \\
\text { desmamada e os pais devem cuidar } \\
\text { da saúde oral do bebê desde cedo. }\end{array}$ & $\begin{array}{l}\text { Falta de consistência } \\
\text { metodológica dos estudos na } \\
\text { associação entre AM e } \\
\text { cáries. Inconsistência na } \\
\text { definição de cáries e AM. }\end{array}$ \\
\hline
\end{tabular}




\section{APENDICE B}

Tabelas 2 - Características das Recomendações por países Características das Recomendações publicadas no Brasil por ordem alfabética

\begin{tabular}{|c|c|c|c|c|c|c|}
\hline Instituição & $\begin{array}{c}\text { Tipo } \\
\text { Instituição }\end{array}$ & Ano & Título & $\begin{array}{c}\text { Tipo } \\
\text { Documento }\end{array}$ & $\begin{array}{c}\text { No. de } \\
\text { Referências }\end{array}$ & $\begin{array}{c}\text { Total RS } \\
\text { Citadas }\end{array}$ \\
\hline $\begin{array}{l}\text { IBFAN Brasil Rede } \\
\text { Internacional em Defesa do } \\
\text { Direito de Amamentar } \\
\text { IBFAN Brasil Rede } \\
\text { Internacional em Defesa do } \\
\text { Direito de Amamentar }\end{array}$ & GOV & 2009 & $\begin{array}{l}\text { Alimentação de lactentes e crianças pequenas em situações de } \\
\text { emergência: manual de orientações para a comunidade, profissionais } \\
\text { de saúde e gestores de programas de assistência comunitária } \\
\text { Benefícios do aleitamento materno e importância dos ácidos graxos de } \\
\text { cadeia longa }\end{array}$ & MCL & 28 & 0 \\
\hline Ministério da Saúde & GOV & 2009 & $\begin{array}{l}\text { Saúde da criança: nutrição infantil. Aleitamento materno e alimentação } \\
\text { complementar }\end{array}$ & MCL & 145 & 2 \\
\hline Ministério da Saúde & GOV & 2002 & Guia alimentar para crianças menores de 2 anos & $\mathrm{R}$ & 295 & 0 \\
\hline Ministério da Saúde & GOV & 2006 & $\begin{array}{l}\text { Guia alimentar para a população brasileira: promovendo a alimentação } \\
\text { saudável }\end{array}$ & $\mathrm{R}$ & 287 & 2 \\
\hline Ministério da Saúde & GOV & 2007 & Promovendo o aleitamento materno & MCL & 0 & 0 \\
\hline Ministério da Saúde & GOV & 2008 & $\begin{array}{l}\text { Iniciativa Hospital Amigo da Criança: Modulo 1- Histórico e } \\
\text { implementação }\end{array}$ & MCL & 43 & 2 \\
\hline Ministério da Saúde & GOV & 2009 & $\begin{array}{l}\text { Iniciativa Hospital Amigo da Criança: Modulo } 2 \text { - fortalecendo e } \\
\text { sustentando a iniciativa Curso para gestores }\end{array}$ & MCL & 257 & 3 \\
\hline Ministério da Saúde & GOV & 2009 & $\begin{array}{l}\text { Iniciativa Hospital Amigo da Criança: Modulo } 3 \text { - Promovendo e } \\
\text { incentivando a amamentação Curso }\end{array}$ & MCL & 88 & 1 \\
\hline Ministério da Saúde & GOV & 2009 & $\begin{array}{l}\text { Iniciativa Hospital Amigo da Criança: Modulo 4- Auto-avaliação e } \\
\text { monitoramento do hospital }\end{array}$ & MCL & 5 & 0 \\
\hline Ministério da Saúde & GOV & 2001 & Como ajudar as mães a amamentar? & MCL & 0 & 0 \\
\hline Ministério da Saúde & GOV & 2011 & $\begin{array}{l}\text { Atenção à saúde do recém-nascido v. } 1 \text { Capítulos: Aleitamento } \\
\text { materno e Dificuldades no aleitamento materno }\end{array}$ & $\mathrm{R}$ & 33 & 2 \\
\hline Ministério da Saúde & GOV & 2011 & $\begin{array}{l}\text { Atenção humanizada ao recém-nascido de baixo peso: método canguru } \\
\text { Manual técnico 2.ed. Mod.3 }\end{array}$ & MCL & 151 & 0 \\
\hline Ministério da Saúde & GOV & 2002 & $\begin{array}{l}\text { Atenção humanizada ao recém-nascido de baixo peso: método } \\
\text { canguru: manual do curso. Sessão } 12 \text {. Nutrição do recém-nascido }\end{array}$ & MCL & 178 & 1 \\
\hline Ministério da Saúde & GOV & 2010 & $\begin{array}{l}\text { Dez passos para uma alimentação saudável: Guia alimentar para } \\
\text { crianças menores de } 2 \text { anos. } 2 \text {.ed. }\end{array}$ & $\mathrm{R}$ & 13 & 0 \\
\hline
\end{tabular}

P2P \& INOVAÇÃO, Rio de Janeiro, v. 5 n. 2, p.133-174, Mar./Ago. 2019. 


\begin{tabular}{|c|c|c|c|c|c|c|}
\hline Instituição & $\begin{array}{c}\text { Tipo } \\
\text { Instituição }\end{array}$ & Ano & Título & $\begin{array}{c}\text { Tipo } \\
\text { Documento }\end{array}$ & $\begin{array}{c}\text { No. de } \\
\text { Referências }\end{array}$ & $\begin{array}{c}\text { Total RS } \\
\text { Citadas }\end{array}$ \\
\hline Ministério da Saúde & $\mathrm{GOV}$ & 2002 & $\begin{array}{l}\text { AIDIPI Atenção integrada as doenças prevalentes na infância. Curso } \\
\text { capacitação. Módulo } 5 \text { Aconselhar a mãe ou o acompanhante }\end{array}$ & MCL & 0 & 0 \\
\hline Ministério da Saúde & GOV & 2004 & $\begin{array}{l}\text { Agenda de compromissos para a saúde integral da criança e redução da } \\
\text { mortalidade infantil }\end{array}$ & $\mathrm{R}$ & 0 & 0 \\
\hline Ministério da Saúde & GOV & 2010 & Política Nacional de Promoção da Saúde. 3 ed. & $\mathrm{P}$ & 7 & 0 \\
\hline Ministério da Saúde & GOV & 2012 & Política Nacional de Alimentação e Nutrição & $\mathrm{P}$ & 31 & 0 \\
\hline Ministério da Saúde & GOV & 2010 & $\begin{array}{l}\text { Alimentação e nutrição para as família dos Programa Bolsa família: } \\
\text { manual para os agentes comunitários de saúde }\end{array}$ & MCL & 7 & 0 \\
\hline Ministério da Saúde & GOV & 2012 & $\begin{array}{l}\text { Saúde da criança: crescimento e desenvolvimento. Cap. } 9 . \\
\text { Alimentação saudável p. 132-156 }\end{array}$ & $\mathrm{R}$ & 65 & 1 \\
\hline Ministério da Saúde & GOV & 1984 & Assistência integral à saúde da criança: ações básicas & $\mathrm{R}$ & 11 & 0 \\
\hline Ministério da Saúde & GOV & 2013 & $\begin{array}{l}\text { Estratégia nacional para promoção do aleitamento materno e } \\
\text { alimentação complementar saudável no SUS - Estratégia Amamenta e } \\
\text { Alimenta Brasil }\end{array}$ & $\mathrm{P}$ & 0 & 0 \\
\hline Ministério da Saúde & GOV & ca1986 & Aleitamento materno e orientação alimentar para o desmame & $\mathrm{R}$ & 0 & 0 \\
\hline $\begin{array}{l}\text { Ministério da Saúde; Rede } \\
\text { Amamenta Brasil }\end{array}$ & GOV & 2009 & Caderno do Tutor Rede Amamenta Brasil & MCL & 36 & 0 \\
\hline $\begin{array}{l}\text { Sociedade Brasileira de } \\
\text { Pediatria }\end{array}$ & AP & 2012 & $\begin{array}{l}\text { Manual de Orientação do Departamento de Nutrologia: alimentação do } \\
\text { lactente ao adolescente }\end{array}$ & MCL & 22 & 0 \\
\hline
\end{tabular}

Tabela 3 - Características das RO publicadas nos EUA por ordem alfabética

\begin{tabular}{|c|c|c|c|c|c|c|}
\hline Instituição & $\begin{array}{c}\text { Tipo } \\
\text { Instituição }\end{array}$ & Ano & Título & $\begin{array}{c}\text { Tipo } \\
\text { Documento }\end{array}$ & $\begin{array}{l}\text { No. de } \\
\text { Ref }\end{array}$ & $\begin{array}{c}\text { Total } \\
\text { RS } \\
\text { Citadas }\end{array}$ \\
\hline $\begin{array}{l}\text { Academy of Breastfeeding } \\
\text { Medicine }\end{array}$ & AP & 2011 & $\begin{array}{l}\text { Breastfeeding the late preterm infant (34 0/7 to } 36 \text { 6/7 weeks gestation): ABM } \\
\text { clinical protocol }\end{array}$ & $\mathrm{R}$ & 0 & 0 \\
\hline $\begin{array}{l}\text { Academy of Breastfeeding } \\
\text { Medicine }\end{array}$ & AP & 2010 & $\begin{array}{l}\text { Non-pharmacologic management of procedure-related pain in the breastfeeding } \\
\text { infant }\end{array}$ & $\mathrm{R}$ & 53 & 3 \\
\hline $\begin{array}{l}\text { Academy of Breastfeeding } \\
\text { Medicine }\end{array}$ & AP & 2008 & Position on Breastfeeding & $\mathrm{D}$ & 24 & 1 \\
\hline
\end{tabular}

P2P \& INOVAÇÃO, Rio de Janeiro, v. 5 n. 2, p.133-174, Mar./Ago. 2019. 


\begin{tabular}{|c|c|c|c|c|c|c|}
\hline Instituição & $\begin{array}{c}\text { Tipo } \\
\text { Instituição }\end{array}$ & Ano & Título & $\begin{array}{l}\text { Tipo } \\
\text { Documento }\end{array}$ & $\begin{array}{l}\text { No. de } \\
\text { Ref }\end{array}$ & $\begin{array}{l}\text { Total } \\
\text { RS } \\
\text { Citadas }\end{array}$ \\
\hline $\begin{array}{l}\text { Agency of Health Research } \\
\text { Quality }\end{array}$ & GOV & 2007 & Breastfeeding and maternal and infant health outcomes in developed countries & RT & 420 & 16 \\
\hline $\begin{array}{l}\text { American Academy of } \\
\text { Family Physicians }\end{array}$ & AP & 2013 & Breastfeeding, Family Physicians Supporting (Position Paper) & $\mathrm{D}$ & 164 & 1 \\
\hline $\begin{array}{l}\text { American Academy of } \\
\text { Nursing }\end{array}$ & AP & 2011 & $\begin{array}{l}\text { The Surgeon General's call to breastfeeding action-policy and practice implications } \\
\text { for nurses }\end{array}$ & $\mathrm{R}$ & 14 & 0 \\
\hline $\begin{array}{l}\text { American Academy of } \\
\text { Pediatric Dentistry }\end{array}$ & AP & 2012 & Policy on dietary recommendations for infants, children, and adolescents & $\mathrm{P}$ & 36 & 0 \\
\hline $\begin{array}{l}\text { American Academy of } \\
\text { Pediatrics }\end{array}$ & AP & 2012 & Breastfeeding and the use of Human Milk: policy statement & $\mathrm{P}$ & 151 & 7 \\
\hline $\begin{array}{l}\text { American Academy of } \\
\text { Pediatrics }\end{array}$ & AP & 2011 & $\begin{array}{l}\text { Sudden infant death syndrome and other sleep-related infant deaths: Expansion of } \\
\text { recommendations for a safe infant sleeping environment }\end{array}$ & $\mathrm{P}$ & 65 & 1 \\
\hline $\begin{array}{l}\text { American Academy of } \\
\text { Pediatrics }\end{array}$ & AP & 2008 & $\begin{array}{l}\text { Effects of early nutritional interventions on the development of atopic disease in } \\
\text { infants and children: the role of maternal dietary restriction, breastfeeding, and } \\
\text { timing of introduction of complementary food (Cap Breastfeeding) }\end{array}$ & RT & 18 & 3 \\
\hline $\begin{array}{l}\text { American College of Nurse- } \\
\text { Midwives }\end{array}$ & AP & 2011 & Position statement & $\mathrm{D}$ & 0 & 0 \\
\hline $\begin{array}{l}\text { American College of } \\
\text { Obstetricians and } \\
\text { Gynecologist }\end{array}$ & AP & 2007 & Breastfeeding: maternal and infant aspects: Special report from ACOG & $\mathrm{RT}$ & 92 & 0 \\
\hline $\begin{array}{l}\text { American Dietetic } \\
\text { Association }\end{array}$ & AP & 2009 & $\begin{array}{l}\text { Position of the American Dietetic Association: promoting and supporting } \\
\text { breastfeeding }\end{array}$ & $\mathrm{D}$ & 141 & 5 \\
\hline $\begin{array}{l}\text { American Heart Association; } \\
\text { American Stroke } \\
\text { Association }\end{array}$ & AP & 2012 & $\begin{array}{l}\text { Breastfeeding health benefits for mother and child: promoting sound lactation } \\
\text { policies in the U.S. }\end{array}$ & $\mathrm{D}$ & 13 & 0 \\
\hline $\begin{array}{l}\text { American Public Health } \\
\text { Association }\end{array}$ & AP & 2013 & An update to A Call to Action on Breastfeeding: a fundamental public health issue & $\mathrm{P}$ & 52 & 2 \\
\hline $\begin{array}{l}\text { Association of Women's } \\
\text { Health, Obstetric and } \\
\text { Neonatal Nurses }\end{array}$ & AP & 2007 & Breastfeeding: Position statement & $\mathrm{D}$ & 0 & 0 \\
\hline Baby-Friendly USA & OI & 2010 & $\begin{array}{l}\text { Baby-Friendly Hospital Initiative Section. Guidelines and evaluation criteria for } \\
\text { facilities seeking baby-friendly designation }\end{array}$ & $\mathrm{R}$ & 11 & 1 \\
\hline $\begin{array}{l}\text { Centers for Disease Control } \\
\text { and Prevention }\end{array}$ & GOV & 2013 & The CDC Guide to strategies to support breastfeeding mothers and babies & $\mathrm{R}$ & 90 & 1 \\
\hline $\begin{array}{l}\text { Centers for Disease Control } \\
\text { and Prevention }\end{array}$ & GOV & 2005 & The CDC guide to breastfeeding interventions & $\mathrm{R}$ & 65 & 0 \\
\hline
\end{tabular}

P2P \& INOVAÇÃO, Rio de Janeiro, v. 5 n. 2, p.133-174, Mar./Ago. 2019. 


\begin{tabular}{|c|c|c|c|c|c|c|}
\hline Instituição & $\begin{array}{c}\text { Tipo } \\
\text { Instituição }\end{array}$ & Ano & Título & $\begin{array}{l}\text { Tipo } \\
\text { Documento }\end{array}$ & $\begin{array}{l}\text { No. de } \\
\text { Ref }\end{array}$ & $\begin{array}{c}\text { Total } \\
\text { RS } \\
\text { Citadas } \\
\end{array}$ \\
\hline $\begin{array}{l}\text { Executive Office of the } \\
\text { President of the United } \\
\text { States }\end{array}$ & GOV & 2010 & $\begin{array}{l}\text { Solving the problem of childhood obesity within a generation. Cap. Early childhood } \\
\text { breastfeeding }\end{array}$ & $\mathrm{R}$ & 338 & 3 \\
\hline Institute of Medicine & OFI & 2011 & Early childhood obesity prevention policies & $\mathrm{R}$ & 0 & 0 \\
\hline $\begin{array}{l}\text { National Association of } \\
\text { Neonatal Nurses NANN }\end{array}$ & AP & 2009 & $\begin{array}{l}\text { The use of human milk and breastfeeding in the neonatal intensive care unit: } \\
\text { position statement }\end{array}$ & $\mathrm{D}$ & 59 & 0 \\
\hline $\begin{array}{l}\text { National Association of } \\
\text { Pediatric Nurse Practitioners }\end{array}$ & AP & 2007 & NAPNAP position statement on breastfeeding & $\mathrm{D}$ & 10 & 0 \\
\hline $\begin{array}{l}\text { National Center for } \\
\text { Education in Maternal and } \\
\text { Child Health }\end{array}$ & GOV & 1997 & $\begin{array}{l}\text { A review of the medical benefits and contraindications to breastfeeding in the } \\
\text { united states }\end{array}$ & $\mathrm{R}$ & 142 & 0 \\
\hline $\begin{array}{l}\text { National Perinatal } \\
\text { Association }\end{array}$ & AP & 2003 & Breastfeeding Position Statement & $\mathrm{D}$ & 0 & 0 \\
\hline National WIC Association & OFI & 2004 & Breastfeeding promotion and support in the WIC Program: position paper & $\mathrm{D}$ & 80 & 0 \\
\hline $\begin{array}{l}\text { U.S. Depart of Health and } \\
\text { Human Services }\end{array}$ & GOV & 2000 & HHS Blueprint for Action on Breastfeeding & $\mathrm{R}$ & 175 & 2 \\
\hline $\begin{array}{l}\text { U.S. Depart of Health and } \\
\text { Human Services - US Surgeon } \\
\text { General }\end{array}$ & GOV & 2011 & Call to action to support breastfeeding & $\mathrm{R}$ & 298 & 6 \\
\hline $\begin{array}{l}\text { United States Breastfeeding } \\
\text { Committee }\end{array}$ & OFI & 2010 & Statement on Breastfeeding as critical strategy for obesity prevention & $\mathrm{D}$ & 7 & 0 \\
\hline $\begin{array}{l}\text { United States Breastfeeding } \\
\text { Committee }\end{array}$ & OFI & 2002 & Benefits of Breastfeeding & $\mathrm{D}$ & 20 & 4 \\
\hline $\begin{array}{l}\text { United States Breastfeeding } \\
\text { Committee }\end{array}$ & OFI & 2000 & Statement on exclusive breastfeeding & $\mathrm{R}$ & 2 & 0 \\
\hline $\begin{array}{l}\text { United States Preventive } \\
\text { Services Task force }\end{array}$ & GOV & 2008 & $\begin{array}{l}\text { Primary care interventions to promote breastfeeding: U.S. Preventive Services Task } \\
\text { force recommendation statement. }\end{array}$ & $\mathrm{R}$ & 11 & 0 \\
\hline
\end{tabular}

P2P \& INOVAÇÃO, Rio de Janeiro, v. 5 n. 2, p.133-174, Mar./Ago. 2019. 
Tabela 4 - Características das RO publicadas no Canadá por ordem alfabética

\begin{tabular}{|c|c|c|c|c|c|c|}
\hline Instituição & $\begin{array}{c}\text { Tipo } \\
\text { Instituição }\end{array}$ & Ano & Título & $\begin{array}{c}\text { Tipo } \\
\text { Documento }\end{array}$ & $\begin{array}{c}\text { No. de } \\
\text { Referências }\end{array}$ & $\begin{array}{l}\text { Total SR } \\
\text { Citadas } \\
\end{array}$ \\
\hline Breastfeeding Committee for Canada & GOV & 2011 & $\begin{array}{l}\text { The WHO } 10 \text { steps to successful breastfeeding (1989) } \\
\text { and the interpretation for Canadian practice }\end{array}$ & $\mathrm{R}$ & 17 & 0 \\
\hline $\begin{array}{l}\text { Canadian Paediatric Society. Nutrition and } \\
\text { Gastroenterology Committee }\end{array}$ & $\mathrm{AP}$ & 2012 & $\begin{array}{l}\text { The Baby-Friendly Initiative: protecting, } \\
\text { promoting and supporting breastfeeding }\end{array}$ & $\mathrm{R}$ & 60 & 2 \\
\hline Canadian Paediatric Society. Nutrition Committee & $\mathrm{AP}$ & 1995 & Nutrition needs and feeding of premature infants & $\mathrm{R}$ & 156 & 0 \\
\hline Canadian Pediatric Society & AP & 2005 & $\begin{array}{l}\text { Exclusive breastfeeding should continue to six } \\
\text { month }\end{array}$ & $\mathrm{R}$ & 10 & 1 \\
\hline Health Canada & GOV & 2004 & $\begin{array}{l}\text { Exclusive breastfeeding duration } 2004 \text { Health } \\
\text { Canada Recommendation }\end{array}$ & $\mathrm{R}$ & 15 & 1 \\
\hline $\begin{array}{l}\text { Health Canada; Canadian Paediatric Society; } \\
\text { Dietitians of Canada; Breastfeeding Committee for } \\
\text { Canada }\end{array}$ & GOV; AP & 2013 & $\begin{array}{l}\text { Nutrition for healthy term infants: } \\
\text { recommendation from birth to six month }\end{array}$ & $\mathrm{R}$ & 206 & 4 \\
\hline $\begin{array}{l}\text { Health Canada; Canadian Paediatric Society; } \\
\text { Dietitians of Canada; Breastfeeding Committee for } \\
\text { Canada }\end{array}$ & GOV; AP & 2013 & $\begin{array}{l}\text { Nutrition for healthy term infants: } \\
\text { recommendation from six to } 24 \text { month }\end{array}$ & $\mathrm{R}$ & 204 & 1 \\
\hline
\end{tabular}

AP = Associação ou sociedade de profissionais de saúde; GOV= Instituição governamental; R= Recomendação

Tabela 5 - Características das RO publicas por organismos internacionais por ordem alfabética

\begin{tabular}{|c|c|c|c|c|c|}
\hline Instituição & Ano & Título & $\begin{array}{c}\text { Tipo } \\
\text { Documento }\end{array}$ & $\begin{array}{c}\text { No. de } \\
\text { Referências }\end{array}$ & $\begin{array}{c}\text { Total SR } \\
\text { Citadas } \\
\end{array}$ \\
\hline $\begin{array}{l}\text { The Baby-Friendly Hospital Initiative for } \\
\text { Neonatal Wards NEO-BFHI }\end{array}$ & 2013 & $\begin{array}{l}\text { Three guiding principles and ten steps to protect, promote and } \\
\text { support breastfeeding }\end{array}$ & $\mathrm{R}$ & 224 & 1 \\
\hline International Confederation of Midwives & 2011 & Breastfeeding & $\mathrm{D}$ & 10 & 0 \\
\hline International Council of Nurses & 2013 & Position statement Breastfeeding & $\mathrm{D}$ & 9 & 0 \\
\hline International Lactation Consultant Association & 2005 & Clinical guideline for the establishment of exclusive breastfeeding & $\mathrm{R}$ & 258 & 2 \\
\hline International Lactation Consultant Association & 2000 & Position paper on infant feeding & $\mathrm{D}$ & 283 & 0 \\
\hline International Lactation Consultant Association & 1999 & $\begin{array}{l}\text { Evidence-based guidelines for breastfeeding management during the } \\
\text { first fourteen days }\end{array}$ & $\mathrm{R}$ & 109 & 0 \\
\hline International Pediatric Association & 2009 & $\begin{array}{l}\text { Support for breast feeding from the International Pediatric } \\
\text { Association }\end{array}$ & $\mathrm{D}$ & 0 & 0 \\
\hline
\end{tabular}

P2P \& INOVAÇÃO, Rio de Janeiro, v. 5 n. 2, p.133-174, Mar./Ago. 2019. 


\begin{tabular}{|c|c|c|c|c|c|}
\hline Instituição & Ano & Título & $\begin{array}{c}\text { Tipo } \\
\text { Documento }\end{array}$ & $\begin{array}{c}\text { No. de } \\
\text { Referências }\end{array}$ & $\begin{array}{l}\text { Total SR } \\
\text { Citadas }\end{array}$ \\
\hline Pan American Health Organization & 2013 & $\begin{array}{l}\text { Beyond survival: integrated delivery care practices for long-term } \\
\text { maternal and infant nutrition } 2 . \text { ed. }\end{array}$ & $\mathrm{R}$ & 147 & 1 \\
\hline Pan American Health Organization & 2002 & $\begin{array}{l}\text { Cuantificación de los beneficios de la lactancia materna: reseña de la } \\
\text { evidencia }\end{array}$ & $\mathrm{R}$ & 186 & 2 \\
\hline $\begin{array}{l}\text { Unicef; WHO; Unesco; UNFPA; UNDP; } \\
\text { UNAIDS; WFP; WB }\end{array}$ & 2010 & Facts for life & $\mathrm{R}$ & 0 & 0 \\
\hline United Nations Children's Fund & 2009 & $\begin{array}{l}\text { Baby-Friendly Hospital Initiative Section 3: Breastfeeding } \\
\text { promotion and support in a Baby-friendly hospital: a 20-hour course } \\
\text { for maternity staff }\end{array}$ & MCL & 38 & 1 \\
\hline Wellstart International & 2013 & Lactation management self-study modules Level I 4thed & MCL & 79 & 2 \\
\hline $\begin{array}{l}\text { World Alliance for Breastfeeding Action - } \\
\text { WABA }\end{array}$ & 2008 & $\begin{array}{l}\text { Protecting, promoting and supporting continued breastfeeding from } \\
6-24+\text { month: issues, politics, policies and action }\end{array}$ & $\mathrm{D}$ & 16 & 0 \\
\hline World Health Organization & 2013 & $\begin{array}{l}\text { Essential nutrition actions: improving maternal, newborn, infant and } \\
\text { young child health and nutrition }\end{array}$ & $\mathrm{R}$ & 381 & 3 \\
\hline World Health Organization & 2013 & $\begin{array}{l}\text { WHO Recommendations on postnatal care of the mother and } \\
\text { newborn }\end{array}$ & $\mathrm{R}$ & 120 & 0 \\
\hline World Health Organization & 2013 & $\begin{array}{l}\text { Guidelines on maternal, newborn, child and adolescent health. } \\
\text { Recommendations on child health }\end{array}$ & $\mathrm{R}$ & 49 & 0 \\
\hline World Health Organization & 2013 & $\begin{array}{l}\text { Guidelines on maternal, newborn, child and adolescent health. } \\
\text { Recommendations on newborn health }\end{array}$ & $\mathrm{R}$ & 4 & 0 \\
\hline World Health Organization & 2011 & $\begin{array}{l}\text { Statement exclusive breastfeeding for six months best for babies } \\
\text { everywhere }\end{array}$ & $\mathrm{D}$ & 1 & 1 \\
\hline World Health Organization & 2011 & $\begin{array}{l}\text { Guidelines on optimal feeding on low birth-weight infants in low- } \\
\text { and-middle-income countries }\end{array}$ & $\mathrm{R}$ & 114 & 0 \\
\hline World Health Organization & 2009 & $\begin{array}{l}\text { Infant and young child feeding: Model chapter for textbooks for } \\
\text { medical students and allied health professionals }\end{array}$ & MCL & 192 & 11 \\
\hline World Health Organization & 2007 & $\begin{array}{l}\text { Planning guide for national implementation of the global strategy for } \\
\text { infant and young feeding }\end{array}$ & $\mathrm{R}$ & 37 & 1 \\
\hline World Health Organization & 2006 & $\begin{array}{l}\text { Pregnancy, childbirth, postpartum and newborn care: a guide for } \\
\text { essential practice } 2 \text {. Ed. }\end{array}$ & $\mathrm{R}$ & 0 & 0 \\
\hline World Health Organization & 2006 & Optimal feeding of low-birth-weight infants & RT & 372 & 6 \\
\hline World Health Organization & 2003 & Global strategy for infant and young child feeding & $\mathrm{R}$ & 0 & 0 \\
\hline World Health Organization & 2002 & Infant and young child nutrition & $\mathrm{P}$ & 0 & 0 \\
\hline World Health Organization & 2001 & $\begin{array}{l}\text { Global strategy for infant and young child feeding: The optimal } \\
\text { duration of exclusive breastfeeding }\end{array}$ & $\mathrm{R}$ & 0 & 0 \\
\hline World Health Organization & 1998 & Evidence for the ten step to successful breastfeeding & $\mathrm{R}$ & 228 & 0 \\
\hline
\end{tabular}




\begin{tabular}{|c|c|c|c|c|c|}
\hline Instituição & Ano & Título & $\begin{array}{c}\text { Tipo } \\
\text { Documento }\end{array}$ & $\begin{array}{c}\text { No. de } \\
\text { Referências }\end{array}$ & $\begin{array}{l}\text { Total SR } \\
\text { Citadas }\end{array}$ \\
\hline World Health Organization & 1997 & Breastfeeding: how to support success & $\mathrm{R}$ & 22 & 0 \\
\hline World Health Organization & 1990 & Infant feeding: the physiological basis. Chapter 2: Lactation & $\mathrm{R}$ & 149 & 0 \\
\hline World Health Organization & s.d. & Essential newborn care course & MCL & 14 & 1 \\
\hline $\begin{array}{l}\text { World Health Organization; Food and } \\
\text { Agriculture Organization of the United Nations } \\
\text { (WHO-FAO) }\end{array}$ & 2003 & $\begin{array}{l}\text { Dieta, nutrición y prevención de enfermedades crónicas: consulta } \\
\text { mixta de expertos OMS/FAO (Cap. } 4,5)\end{array}$ & $\mathrm{RT}$ & 528 & 0 \\
\hline $\begin{array}{l}\text { World Health Organization; United Nations } \\
\text { Children's Fund }\end{array}$ & 2013 & Ten step to successful breastfeeding & $\mathrm{R}$ & 0 & 0 \\
\hline $\begin{array}{l}\text { World Health Organization; United Nations } \\
\text { Children's Fund }\end{array}$ & 2009 & $\begin{array}{l}\text { Baby-Friendly Hospital Initiative Section 2: Strengthening and } \\
\text { sustaining the Bay-friendly hospital initiative: a course for decision- } \\
\text { makers }\end{array}$ & MCL & 311 & 6 \\
\hline $\begin{array}{l}\text { World Health Organization; United Nations } \\
\text { Children's Fund }\end{array}$ & 2006 & $\begin{array}{l}\text { Infant and young child feeding counseling: an integrated course } 4 \mathrm{v} \\
+ \text { slides }\end{array}$ & MCL & 40 & 1 \\
\hline $\begin{array}{l}\text { World Health Organization; United Nations } \\
\text { Children's Fund }\end{array}$ & 1993 & Breastfeeding counseling: a training course & MCL & 24 & 0 \\
\hline $\begin{array}{l}\text { World Health Organization; United Nations } \\
\text { Children's Fund }\end{array}$ & 1989 & Protecting, promoting and supporting breast-feeding & $\mathrm{D}$ & 0 & 0 \\
\hline
\end{tabular}

$\mathrm{D}$ = Declaração; MCL = Material para cursos e livros; $\mathrm{P}$ = Política pública; $\mathrm{R}=$ Recomendação; $\mathrm{RT}$ = Relatório téc 


\section{APÊNDICE C \\ Referências das Revisões Sistemáticas incluídas}

1. AKOBENG, A. K. et al. Effect of breast feeding on risk of coeliac disease: a systematic review and meta-analysis of observational studies. Archives of Disease in Childhood, v. 91, n. 1, p. 39-43, 2006.

2. ANDERSON, J. W.; JOHNSTONE, B. M.; REMLEY, D. T. Breast-feeding and cognitive development: a meta-analysis. American Journal of Clinical Nutrition, v. 70, n. 4, p. 525$535,1999$.

3. ARENZ, S. et al. Breast-feeding and childhood obesity - a systematic review. International Journal of Obesity, v. 28, n. 10, p. 1247-1256, 2004.

4. BACHRACH, V. R.; SCHWARZ, E.; BACHRACH, L. R. Breastfeeding and the risk of hospitalization for respiratory disease in infancy: a meta-analysis. Archives of Pediatrics and Adolescent Medicine, v. 157, n. 3, p. 237-243, 2003.

5. BARCLAY, A. R. et al. Systematic review: the role of breastfeeding in the development of pediatric inflammatory bowel disease.The Journal of Pediatrics, v. 155, n. 3, p. 421-426, 2009.

6. DER, G.; BATTY, G. D.; DEARY, I. J. Effect of breast feeding on intelligence in children: prospective study, sibling pairs analysis, and meta-analysis. British Medical Journal, v. 333, n. 7575, p. 945-948, 2006.

7. GDALEVICH, M.; MIMOUNI, D.; MIMOUNI, M. Breast-feeding and the risk of bronchial asthma in childhood: a systematic review with meta-analysis of prospective studies. The Journal of Pediatrics, v. 139, n. 2, p. 261-266, 2001 a.

8. GDALEVICH, M. et al. Breast-feeding and the onset of atopic dermatitis in childhood: a systematic review and meta-analysis of prospective studies. Journal of the American Academy of Dermatology, v. 45, n. 4, p. 520-527, $2001 \mathrm{~b}$.

9. HARDER, T. et al. Duration of breastfeeding and risk of overweight: a meta-analysis. American Journal of Epidemiology, v. 162, n. 5, p. 397-403, 2005.

10. HAUCK, F. R. et al. Breastfeeding and reduced risk of sudden infant death syndrome: a meta-analysis. Pediatrics, v. 128, n. 1, p. 103-110, 2011.

11. HENDERSON, G.; ANTHONY, M. Y.; MCGUIRE, W. Formula milk versus term human milk for feeding preterm or low birth weight infants. The Cochrane Database of Systematic Reviews, n. 4, p. Cd002971, 2001 b.

12. HORTA, B. L. E. A. Evidence on the long-term effects of breastfeeding: systematic reviews and meta-analyses. Geneva: WHO, 2007 
13. KLEMENT, E. et al. Breastfeeding and risk of inflammatory bowel disease: a systematic review with meta-analysis. The American Journal of Clinical Nutrition, v. 80, n. 5, p. 1342-1352, 2004.

14. KRAMER, M.; KAKUMA, R. Optimal duration of exclusive breastfeeding. TheCochrane Database of Systematic Reviews, n. 1, p. CD003517, 2009.

15. KRAMER, M.; KAKUMA, R. The Optimal duration of exclusive breastfeeding: a systematic review.Geneve: WHO, 2002a

16. KRAMER, M.; KAKUMA, R. Optimal duration of exclusive breastfeeding. TheCochrane Database of Systematic Reviews, n. 1, p. Cd003517, 2002 b.

17. KRAMER, M.; KAKUMA, R. The optimal duration of exclusive breastfeeding: a systematic review. Advances in Experimental Medicine and Biology, v. 554, n., p. 63-77, 2004.

18. KWAN, M. L. et al. Breastfeeding and the risk of childhood leukemia: a metaanalysis. Public Health Reports, v. 119, n. 6, p. 521-535, 2004.

19. MARTIN, R. M. et al. Breast-feeding and childhood cancer: a systematic review with metaanalysis. International Journal of Cancer, v. 117, n. 6, p. 1020-1031, 2005a.

20. MARTIN, R. M. et al. Breastfeeding and cardiovascular mortality: the Boyd Orr cohort and a systematic review with meta-analysis. European Heart Journal, v. 25, n. 9, p. 778-786, 2004.

21. MCGUIRE, W.; ANTHONY, M. Y. Donor human milk versus formula for preventing necrotising enterocolitis in preterm infants: systematic review. Archives of Disease in Childhood. Fetal and Neonatal Edition, v. 88, n. 1, p. F11-14, 2003.

22. NORRIS, J. M.; SCOTT, F. W. A meta-analysis of infant diet and insulin-dependent diabetes mellitus: do biases play a role? Epidemiology, v. 7, n. 1, p. 87-92, 1996.

23. OWEN, C. G. et al. The effect of breastfeeding on mean body mass index throughout life: a quantitative review of published and unpublished observational evidence. American Journal of Clinical Nutrition, v. 82, n. 6, p. 1298-1307, 2005a.

24. OWEN, C. G. et al. Does breastfeeding influence risk of type 2 diabetes in later life?:a quantitative analysis of published evidence. American Journal of Clinical Nutrition, v. 84, n. 5, p. 1043-1054, 2006.

25. OWEN, C. G. et al. Effect of breast feeding in infancy on blood pressure in later life: systematic review and meta-analysis. British Medical Journal, v. 327, n. 7425, p. 11891195, 2003.

26. OWEN, C. G. et al. Infant feeding and blood cholesterol: a study in adolescents and a systematic review. Pediatrics, v. 110, n. 3, p. 597-608, 2002. 
27. QUIGLEY, M. A. et al. Formula milk versus donor breast milk for feeding preterm or low birth weight infants. The Cochrane Database of Systematic Reviews, n. 4, p. Cd002971, 2007.

28. SHAH, P. S.; ALIWALAS, L.; SHAH, V. Breastfeeding or breastmilk to alleviate procedural pain in neonates: a systematic review. Breastfeed Medicine, v. 2, n. 2, p. 74-82, 2007.

29. SHAH, P. S.; ALIWALAS, L. I.; SHAH, V. Breastfeeding or breast milk for procedural pain in neonates. The Cochrane Database of Systematic Reviews, n. 3, p. Cd004950, 2006.

30. VALAITIS, R. et al. A systematic review of the relationship between breastfeeding and early childhood caries. Canadian Journal of Public Health, v. 91, n. 6, p. 411-417, 2000 . 